\title{
Trans-dimensional Bayesian inversion of airborne transient EM data from Taylor Glacier, Antarctica
}

\author{
Daniel Blatter, ${ }^{1}$ Kerry Key, ${ }_{1}^{1}$ Anandaroop Ray, ${ }^{2}$ Neil Foley, ${ }^{3}$ Slawek Tulaczyk ${ }^{3}$ and \\ Esben Auken $^{4}$ \\ ${ }^{1}$ Lamont-Doherty Earth Observatory, Columbia University, Palisades, NY 10964, USA. E-mail: daniel.blatter@columbia.edu \\ ${ }^{2}$ Chevron Corporation, Houston, TX 77002, USA \\ ${ }^{3}$ Earth and Planetary Sciences, University of California, Santa Cruz, Santa Cruz, CA 95064, USA \\ ${ }^{4}$ Aarhus University, Department of Geosciences, 8000 Aarhus, Denmark
}

Accepted 2018 June 22. Received 2018 January 17; in original form 2018 June 12

\begin{abstract}
A BSTRACT
Conventional regularized nonlinear inversion methods for estimating electrical conductivity from observed electromagnetic data seek to find a single model that fits the data while minimizing a user-imposed model regularization norm. By contrast, Bayesian sampling techniques produce a large suite of models, all of which fit the data adequately, providing a wealth of statistical information about the model parameters. Importantly, this includes quantitative uncertainty estimates as well as any statistical property of interest. In this work, we apply a Bayesian trans-dimensional Markov chain Monte Carlo scheme to recover subsurface conductivity from airborne transient electromagnetic (TEM) data collected over Taylor Glacier, Antarctica, to image subglacial hydrologic structure. We provide a synthetic model study, followed by inversions of real soundings. Our results identify a zone of conductive, wet sediments beneath the glacier, corroborating interpretations from previous studies that used regularized, smooth inversions. Our results provide, however, the opportunity to examine a rich suite of additional information, including uncertainty estimates on the conductivity within the conductive subglacial layer as well as quantitative estimates of its total conductance. We apply principles of Bayesian information theory for estimating the depth of investigation of the airborne TEM data and apply it to this data set. Additionally, we use the model ensemble to derive estimates of pore fluid resistivity within the conductive layer, with associated uncertainties. Finally, we use Bayesian model studies to explore the range of ice thicknesses and conductive layer thicknesses that could be resolved with ground or airborne TEM data if they had one to two orders of magnitude lower noise levels.
\end{abstract}

Key words: Antarctica.; Inversion; Electromagnetic induction; Statistical methods; Hydrology.

\section{INTRODUCTION}

Electromagnetic (EM) methods use passive or active source EM fields to probe subsurface conductivity structure. Depending on the frequency content of the source fields, the depth of investigation (DOI) can range from hundreds of kilometres to just a few metres.

The past two decades have seen rapid advances in airborne systems. Both frequency domain and transient systems have been developed with a wide range of applications, including ground water mapping (e.g. Siemon et al. 2009) and mineral exploration (e.g. Fountain et al. 2005).

Helicopter transient electromagnetic (TEM) systems consist primarily of a large wire loop slung beneath the helicopter in which a current is pulsed at regular intervals. The primary magnetic field produced by this current induces secondary EM fields whose rate of decay is a nonlinear function of the local subsurface conductivity. These secondary EM fields are measured using a magnetic field receiver also towed beneath the helicopter. Because the strength and duration of the secondary magnetic field depends on the currents induced in the subsurface, the TEM method is primarily sensitive to subsurface conductive rather than resistive structures.

These systems have a number of desirable features, including the ability to collect data with a dense spatial sampling within a relatively short acquisition time frame and nominal depth sensitivity of more than $400 \mathrm{~m}$, depending on conductivity structure (Legault 2015; Sørensen \& Auken 2004). In addition, having the transmitter and receiver collocated makes the data more suitable for 1-D modelling than long-offset soundings. 
The measured fields must be inverted to recover an estimate of subsurface conductivity. The standard inversion technique for TEM data, as well as EM geophysical data in general, relies upon an objective function that is typically the sum of the data misfit between measured and forward modelled data, and a regularization penalty against model roughness and/or difference from a reference model. Gradients in the objective function are then used to attempt to discover the model that minimizes this function. An efficient approach is to invert each airborne TEM sounding separately for a 1-D Earth model, where the 1-D approximation is justified by the relatively narrow sensitivity footprint of the collocated TEM transmitter and receiver system, compared to typical lateral variability of subsurface structures. However, in regions of high spatial heterogeneity, the 1-D assumption breaks down. The resulting 1-D models can be stitched together to form 2-D or 3-D images. Auken \& Christiansen (2004) extend this approach to quasi-2-D models by including smoothness constraints not only with depth but also laterally along the flight path of the helicopter. Viezzoli et al. (2008) produce quasi-3-D models by adding smoothness constraints between all neighbouring 1-D models in any horizontal direction. More physically rigorous 3-D modelling (Newman \& Alumbaugh 1995) and inversion (Cox et al. 2012) algorithms have also been developed and applied to field data (Yang \& Oldenburg 2012). In all of these approaches, the inversion is run until it produces a model that fits the data to within a user specified tolerance-ideally to within the data uncertainty. Other nearby models can also be found by adjusting the data fit tolerance or by adjusting the relative weighting of the model roughness norm, which results in finding one or more additional models that are usually in a neighbourhood of the original best fitting model.

What these methods leave unaddressed is the uncertainty in the estimated model parameters. Most nonlinear inverse problems are non-unique - meaning that, depending on how one chooses to parametrize the Earth's conductivity structure, an infinite number of models may adequately explain the measured data. In fact, regularization must be introduced in the formulation of the objective function precisely to constrain this non-uniqueness and allow the inverse algorithm to converge to a solution. However, it is often the case that estimating parameter uncertainty and/or model nonuniqueness is as important as estimating the parameter values themselves. If, for instance, a geologic interface characterized by strong conductivity contrasts is known to exist at some depth in the subsurface, one might wish to know the range of depths this interface can span while fitting the data to within its uncertainty. Also of interest in EM induction methods is an estimate of the DOI-the maximum depth in the Earth at which the data are still sensitive to the electrical conductivity structure. Approximate methods for estimating the DOI include using a half-space skin depth or the Jacobian sensitivity matrix (e.g. Christiansen \& Auken 2012), yet these fail to take into account the nonlinear sensitivity of the DOI to conductivity structure.

Bayesian sampling-based inverse methods are a class of algorithms that provide an estimate of model parameter uncertainty by generating an ensemble of models - each of which fits the data, and from which statistical properties of the model parameters can be inferred (Mosegaard \& Tarantola 1995). They do not require linearization, nor do they require regularization, and they provide a more robust measure of the DOI. These benefits come at the price of significant, additional computational cost. In this work, we sample the Bayesian posterior probability density function from TEM data using a Markov chain Monte Carlo (MCMC) method based on the Metropolis-Hastings algorithm (Metropolis et al. 1953; Hastings 1970).

\section{MODEL PARAMETRIZATION AND FORWARD CALCULATIONS}

Motivated by the reasonableness of the 1-D assumption for this application and the high computational cost of MCMC, we choose a 1-D parametrization of the Earth such that our model consists of $k$ layer interfaces and $k+1$ layers, each with an associated electrical resistivity. The model, then, consists of $k$ interface depths $\mathbf{z}=\left[z_{1}, z_{2}, \ldots z_{k}\right]$ and $k+1$ layer resistivities $\rho=$ $\left[\log \left(\rho_{1}\right), \log \left(\rho_{2}\right), \ldots \log \left(\rho_{k}\right), \log \left(\rho_{k+1}\right)\right]$. The last layer is assumed to be a semi-infinite half-space.

The vertical magnetic field $B_{z}(t)$ produced by the combined effects of the loop source and the resulting ground response is calculated by computing the path integral of point horizontal electric dipoles distributed over the eight wire segments of the octagonal transmitter loop. The point dipole fields were first computed in the frequency domain using a variant of the 1-D code described by Key (2009) and were subsequently transformed to the time domain using the digital filter method (e.g. Newman et al. 1986; Key 2012). The path integrals were efficiently computed using Gauss-Legendre quadrature. Because the current in the transmitter loop cannot be switched instantaneously, it is also necessary to model correctly the finite duration of the ramp-on and ramp-off in the transmitter current, which for the particular TEM system considered here were $8 \times 10^{-4}$ and $6.5 \times 10^{-6} \mathrm{~s}$, respectively. Here we use the efficient convolution method of Fitterman \& Anderson (1987) to simulate the ramp-on and -off effects. The data was modelled at time gates of roughly $10^{-5}$ to $10^{-2} \mathrm{~s}$, depending on the system noise. All responses are normalized by the transmitter moment, which is the product of the loop area $\left(499 \mathrm{~m}^{2}\right)$ and the current $(500 \mathrm{~A})$.

\section{BAYESIAN INVERSION FRAMEWORK}

Bayesian probability describes how additional information (provided by measured data) modifies a priori assumptions to generate a posteriori probabilities. For an accessible discussion, see Scales \& Snieder (1997). Bayesian information is summarized in Bayes' rule

$p(\mathbf{m} \mid \mathbf{d}) \propto p(\mathbf{d} \mid \mathbf{m}) p(\mathbf{m})$

where the variables to the left of $\mid$ are conditional on those to the right. In other words, $p(a \mid b)$ is the probability of $a$ given that $b$ is known. $\mathbf{m}$ is a vector of parameters that constitute our model of the Earth (in our case, $k, \mathbf{z}$ and $\rho$ ), while $\mathbf{d}$ is the vector of observed data. We will discuss each term in (1) individually.

The $p(\mathbf{d} \mid \mathbf{m})$ term on the right hand side is called the model likelihood, and is a measure of data fit. The likelihood is the probability that the modelled data differ from the measured data purely due to random measurement error. Details on the specific form of the likelihood can be found in Appendix A. The $p(\mathbf{m})$ term on the right hand side of (1) is the a priori probability density function of the model parameters, known simply as the prior. It represents all the information known and assumptions made about the model-independent of the measured data.

Finally, the product of the prior and the likelihood yields the $a$ posteriori probability density function (PDF) of the model parameters, known as the posterior and written as $p(\mathbf{m} \mid \mathbf{d})$. Each element, $\mathbf{m}$, of the model space has an associated probability, conditional on the measured data. Obtaining a good approximation to the posterior is the objective of sampling-based Bayesian inverse methods, as the posterior contains all the information about the model parameters provided by the measured data and prior information. If the 
posterior closely resembles the prior, then the data have provided little new information, while any major differences indicate that the data have modified our prior assumptions. For nonlinear inverse problems we must draw samples from the posterior, as its analytical form is unknown. Once enough samples have been drawn, they can be used to approximate the posterior PDF.

In the next section we review our Bayesian sampling method: a trans-dimensional MCMC algorithm using the MetropolisHastings criterion.

\subsection{Trans-dimensional MCMC}

One algorithm for drawing samples from the posterior that has seen widespread use across many disciplines due to its efficiency, robustness, and simplicity is MCMC, which samples the model space using a kind of guided random walk. The algorithm produces an ensemble of models that converges to the posterior as the number of samples in the ensemble grows (for a helpful discussion of MCMC, see Gilks et al. 1995).

The particular sampling method used here is the MetropolisHastings algorithm (Metropolis et al. 1953; Hastings 1970), in which the Markov chain (the model ensemble) grows iteratively by adding a new model to the end of the chain at each step. A proposal model $\mathbf{m}^{\prime}$ is generated by drawing from a proposal distribution $q\left(\mathbf{m}^{\prime} \mid \mathbf{m}\right)$. The notation for the proposal indicates that this is done by perturbing the last model in the chain, $\mathbf{m}$, in some way. Then, either $\mathbf{m}^{\prime}$ is accepted as the newest member of the chain, or $\mathbf{m}$ is retained (and thus appears in the chain twice in a row). Whether or not $\mathbf{m}^{\prime}$ is accepted depends upon the acceptance probability $\alpha\left(\mathbf{m}^{\prime} \mid \mathbf{m}\right)$, which will be discussed shortly.

Green (1995) extended the Metropolis-Hastings algorithm to allow the proposed model, $\mathbf{m}^{\prime}$, to have a different number of model parameters than the model, $\mathbf{m}$, from which it was derived. This trans-dimensionality is accomplished by means of the so-called reversible jumps and allows the number of model parameters to be itself a model parameter. Both the number of layer interfaces and their depths are variable in our trans-dimensional MCMC algorithm. This additional flexibility is an advantage in that the appropriate number of interfaces and their locations are typically not known a priori. Instead, the trans-dimensional MCMC algorithm allows the information provided by the data to drive the choice of $k, \rho$ and $\mathbf{z}$. In this process, for a given data fit, models with fewer layers are preferred by our algorithm over models with more parameters due to the natural parsimony implicit in Bayes' rule (MacKay 2003; Malinverno 2002). Bayesian parsimony does not guarantee that only the models with the minimum possible number of interfaces/layers will be accepted in the model ensemble. Rather, all else being equal, Bayesian inversion prefers models with fewer parameters. While reversible jump MCMC technically allows jumps of any size (from $k$ model parameters to $k^{\prime}$, where both $k$ and $k^{\prime}$ are arbitrary integers), we implement here the 'birth-death' scheme (Geyer \& Moller 1994), whereby each successive model in the chain may have one more layer interface, one fewer, or the same number of interfaces.

Trans-dimensional MCMC has been applied successfully to a wide range of problems in the Earth sciences. In seismology, it has been applied to acoustic seabed reflectivity (Dettmer et al. 2010), receiver functions (Agostinetti \& Malinverno 2010), microtremor (Dettmer et al. 2012), surface wave tomography (Saygin et al. 2015; Galetti et al. 2016) and full waveform inversion (Ray et al. 2016). In electromagnetics, it has been applied to frequency domain airborne EM (e.g. Minsley 2011), time domain airborne EM (e.g. Hawkins et al. 2017; Brodie \& Sambridge 2012) and controlled-source electromagnetics (e.g. Ray \& Key 2012; Ray et al. 2014; Gehrmann et al. 2015); Luo (2010) applied it to gravity data, while Laine \& Tamminen (2008) applied it to modelling atmospheric aerosols.

The acceptance probability, $\alpha\left(\mathbf{m}^{\prime} \mid \mathbf{m}\right)$, is the key to ensuring that the Markov chain converges to the posterior since it draws samples according to the posterior's density. Convergence is guaranteed if $\alpha$ is chosen to be:

$$
\begin{aligned}
\alpha\left(\mathbf{m}^{\prime} \mid \mathbf{m}\right)= & \min [1, \quad \text { likelihood ratio } \times \text { prior ratio } \\
& \times \text { proposal ratio } \times|J|] \\
= & \min \left[1, \quad\left(\frac{p\left(\mathbf{d} \mid \mathbf{m}^{\prime}\right)}{p(\mathbf{d} \mid \mathbf{m})}\right)^{1 / T} \frac{p\left(\mathbf{m}^{\prime}\right)}{p(\mathbf{m})} \frac{q\left(\mathbf{m} \mid \mathbf{m}^{\prime}\right)}{q\left(\mathbf{m}^{\prime} \mid \mathbf{m}\right)} \times|J|\right] .
\end{aligned}
$$

The matrix $J$ is the Jacobian of the jump from $\mathbf{m}$ to $\mathbf{m}^{\prime}$ (not to be confused with the forward sensitivity matrix of partial first derivatives). $J$ in a sense normalizes out the difference in volume between the spaces inhabited by $\mathbf{m}$ and $\mathbf{m}^{\prime}$. For the types of trans-dimensional jumps used in this paper, $|J|=1$ (Agostinetti \& Malinverno 2010), so this term can be ignored from here on. $T$ is a tempering value $\geq 1$, explained in detail in the next section. The logic of this definition of the acceptance probability can be understood intuitively, in the case where the prior is uniform and the proposal is symmetric, since $\alpha$ simplifies to just the ratio of the likelihoods. Thus, if the likelihood (i.e. the data fit) improves by moving from $\mathbf{m}$ to $\mathbf{m}^{\prime}$, then the move will always be accepted. If the likelihood at $\mathbf{m}^{\prime}$ is lower than at $\mathbf{m}$, the move can still be accepted, but with probability equal to the ratio of the likelihoods (which will be less than one). This will ensure that the high probability regions of the model space are more densely sampled than lower probability regions. Further details concerning the specific form of the proposal and the acceptance probability can be found in Appendix A.

\subsection{Parallel tempering}

One limitation of Bayesian sampling methods is the difficulty of effectively sampling a high dimensional model space in a feasible amount of time. In order to accelerate convergence of the Markov chains to the high probability regions of the model space, we implemented a technique known as parallel tempering (PT) (Geyer 1991; Falcioni \& Deem 1999) which was first developed for use in molecular dynamics (Swendsen \& Wang 1987) but has more recently been applied to inverse problems in geophysics (e.g. Dosso et al. 2012; Ray et al. 2013).

The key idea behind PT is the augmentation of the model space with a new dimension, represented by the variable $T$. If we define

$\pi(\mathbf{m}, T)=C^{-1} p(\mathbf{d} \mid \mathbf{m})^{1 / T} p(\mathbf{m})$,

where $C$ is the normalizing constant, then the role of $T$ (or "temperature") becomes apparent. For large values of T, the likelihood term goes to one and $\pi$ becomes the prior. For $T=1$, on the other hand, $\pi$ becomes the posterior. For intermediate values, $\mathrm{T}$ has the effect of tempering the impact of the likelihood, thus allowing Markov chains with temperature $T>1$ to sample the model space more freely because they are more tolerant of models at higher values of misfit. This permits these warmer chains to escape local maxima (in terms of posterior probability) and more efficiently and completely sample the model space.

Of course, the misfit space one wishes to sample is that of $T=1$. The tactic employed in PT, then, is to run multiple chains in parallel 


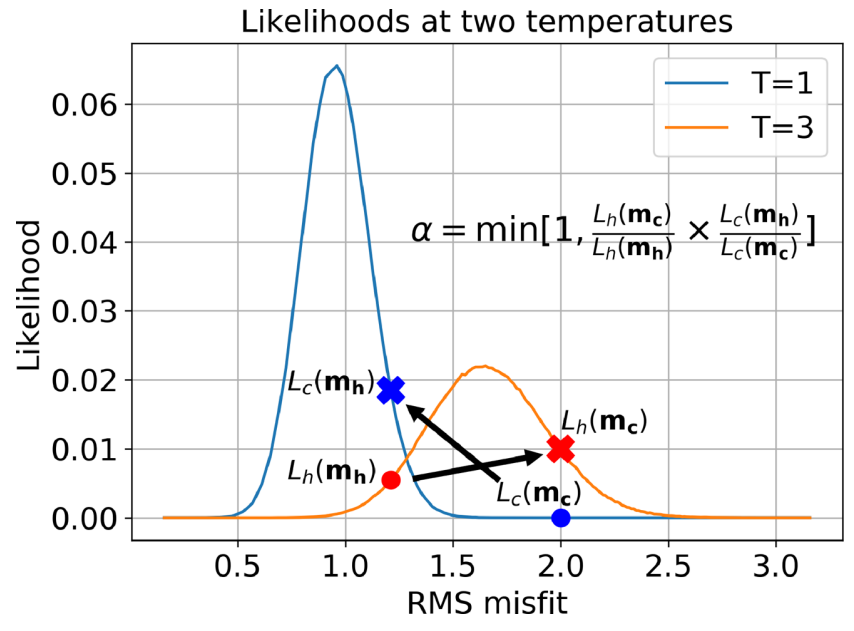

Figure 1 Model swapping between chains accelerates convergence of untempered $(T=1)$ Markov chains to the low-misfit regions of model space. Two chains-at $T=3$ (red) and $T=1$ (blue)-swap models with probability $\alpha$, where $L_{\mathrm{c}}$ and $L_{\mathrm{h}}$ (plotted in blue and red, respectively) are the likelihood functions for the untempered (cold) and tempered (hot) misfit spaces. The dots represent the pre-swap state of each, while the crosses represent their post-swap states. Because the untempered likelihood is extremely low at $\mathbf{m}_{\mathrm{c}}$, the ratio on the right in the swap probability, $\alpha$, dominates, and a swap is certain to occur. As a result, the effect of PT is to facilitate swaps that accelerate the convergence of the colder chains to the lower misfit regions of model space.

at different temperatures, and to allow them to swap models at each step. This can be done such that the swapping does not bias the posterior distribution for the $T=1$ chain. Writing the likelihood for the $i^{\text {th }}$ temperature as $L_{i}(\mathbf{m})=p(\mathbf{d} \mid \mathbf{m})^{1 / T_{i}}$, the swap probability is

$\alpha_{\text {swap }}(i, j)=1 \wedge\left[\frac{L_{i}\left(\mathbf{m}_{j}\right)}{L_{i}\left(\mathbf{m}_{i}\right)} \times \frac{L_{j}\left(\mathbf{m}_{i}\right)}{L_{j}\left(\mathbf{m}_{j}\right)}\right]$,

with models $\mathbf{m}_{i}$ and $\mathbf{m}_{j}$ in the $i^{\text {th }}$ and $j^{\text {th }}$ chains, respectively.

Markov chains exploring the untempered misfit space often find it difficult to converge from regions of model space that fit the data moderately well - a root-mean-square (RMS) misfit of, say, 2.0to regions that fit the data well (an RMS of roughly 1.0). This is primarily because, by RMS 2.0 , fine-scale changes are needed to improve the fit to the data, while the perturbations at each step in the MCMC algorithm are random. Fig. 1 shows how PT is effective at accelerating untempered chains through this phase of convergence. In Fig. 1, the untempered likelihood and a tempered likelihood (for $T=3$ ) are plotted as a function of RMS misfit. The hot chain has found a model, $\mathbf{m}_{\mathbf{h}}$, that fits the data better than the cold chain, at model state $\mathbf{m}_{\mathbf{c}}$ (RMS misfits of 1.2 and 2.0, respectively). This is precisely the scenario where swapping models would accelerate the convergence of the untempered chain to the regions of high likelihood. If the two chains propose to swap models, the rightmost ratio of $\alpha_{\text {swap }}$ will be very large, guaranteeing a swap so long as there is some non-trivial overlap between the two likelihood functions. That is, so long as the left-most ratio of eq. (5), and on the left in Fig. 1, is not $\ll 1$. The idea is to construct a ladder of temperatures such that there is a 'Goldilocks zone' of overlap between each pair of adjacent likelihood functions - not too little overlap, which makes the likelihood of a swap too low; nor too much overlap, which renders the adjacent chains largely redundant. A well-constructed temperature ladder ensures that as the warmer chains explore the model space, any lower-RMS misfit models they find will be quickly swapped down the temperature ladder to the coldest chains.

In summary, PT enhances the robustness and efficiency with which the $T=1$ Markov chain samples the model space, leading to a better approximation of the posterior distribution in fewer steps. For a more thorough discussion of PT with numerical examples, see Sambridge (2013). In Appendix B, we provide a short pseudo-code that illustrates the flow of the complete algorithm.

\section{SYNTHETIC EXAMPLE}

To demonstrate the efficacy of our MCMC algorithm, we conducted a synthetic test. Synthetic data were generated by computing the forward response of a three-layer subglacial-conductor model (Fig. 2; left panel, in blue), then adding random noise. We used a simplified noise model consisting of 5 per cent relative Gaussian noise applied to all time gates, plus absolute Gaussian noise at a noise floor equal to $10^{-14} \mathrm{~V} \mathrm{Am}^{-4}$ (Fig. 2, right panel, in blue). Synthetic data below the noise floor were removed. The transmitter loop was flown $35 \mathrm{~m}$ above the surface, and the data were normalized by the transmitter moment.

The upper $150 \mathrm{~m}$ of the model are resistive $(10000 \mathrm{ohm} \mathrm{m}$, consistent with a layer of ice), beneath which is a $50 \mathrm{~m}$ thick conductive layer $(10 \mathrm{ohm} \mathrm{m})$. The final layer is a resistive $(1000 \mathrm{ohm} \mathrm{m})$ halfspace. We also computed the forward response of a background model which is identical in the upper $150 \mathrm{~m}$, but which transitions to the $1000 \mathrm{ohm}$ m basement immediately thereafter (shown in red in both panels of Fig. 2) for comparison. The larger response of the subglacial conductor model is due to the presence of the conductor, which sustains larger induced currents than the background model.

We ran the MCMC algorithm with this synthetic data set using PT with eight chains (three at $T=1$, using the temperature ladder $T=[1,1,1,1.15,1.32,1.52,1.74,2])$ for 260000 iterations. The total computation consisted then of $2080000(8 \times 260000)$ forward computations, all of which were done serially in MATLAB. Each computation took roughly $0.2 \mathrm{~s}$, for a total computation time of roughly $5 \mathrm{~d}$. This could be reduced further by a factor of 8 (the number of chains) by using parallel computing constructs to run the chains in parallel using 8 processing cores. Further speed ups could be accomplished by computing each forward model response in parallel, given the independent linear systems associated with each frequency and wave-number required by the 1-D TEM calculations. If both the PT chains and 1-D soundings were computed in parallel, the algorithm could easily scale to use hundreds of processors and obtain a significant speedup over the time required for the serial version we implemented here. Inverting an entire airborne TEM data set in this way could, in theory, be accomplished rapidlyalbeit requiring significant computational resources.

We verified convergence using plots of RMS misfit and chain swap rate as a function of model number in the chains (Fig. 3). We combined the final 200000 samples (the post-burn-in samples) from the three $T=1$ chains, and computed an estimate of the posterior from this ensemble. The final model ensemble from which the posterior was estimated, then, contained 600000 models. Fig. 4(a) shows the estimate of the posterior probability density function of resistivity and depth. Warmer colours indicate higher probability density, while cooler colours indicate lower density. One way of understanding this kind of plot is to view each horizontal slice across the plot as the PDF of resistivity at that particular depth. Red lines indicate the resistivity at which the 5 th and 95 th percentiles occur at that depth. The black dashed line indicates the true model. 

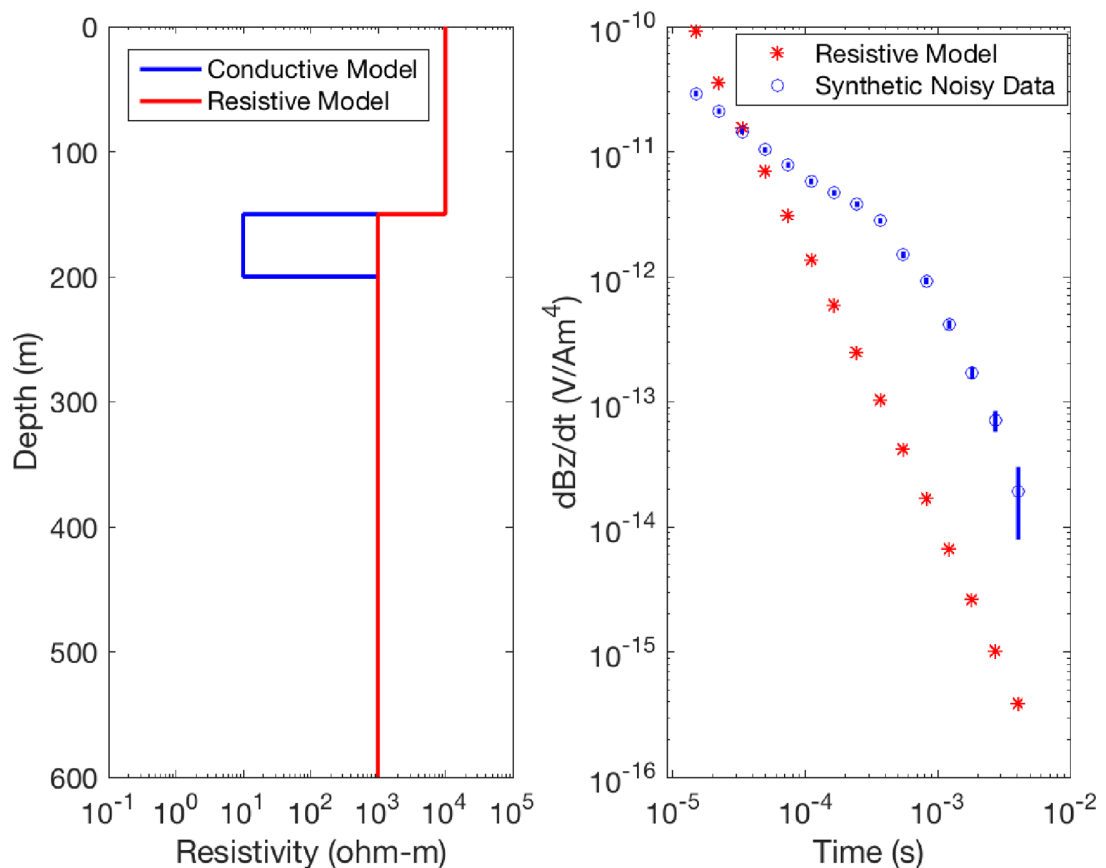

Figure 2 A synthetic subglacial-conductor model, representing $150 \mathrm{~m}$ of resistive ice $\left(10^{4} \mathrm{ohm} \mathrm{m}\right)$ with a $50 \mathrm{~m}$ thick conductive $(10 \mathrm{ohm} \mathrm{m})$ wet sediment channel overlying a half-space $\left(10^{3} \mathrm{ohm} \mathrm{m}\right)$, is plotted in blue on the left panel, and its corresponding forward response, with added noise, is also plotted in blue on the right panel. For reference, a resistive two-layer background model and its response are similarly plotted in red.
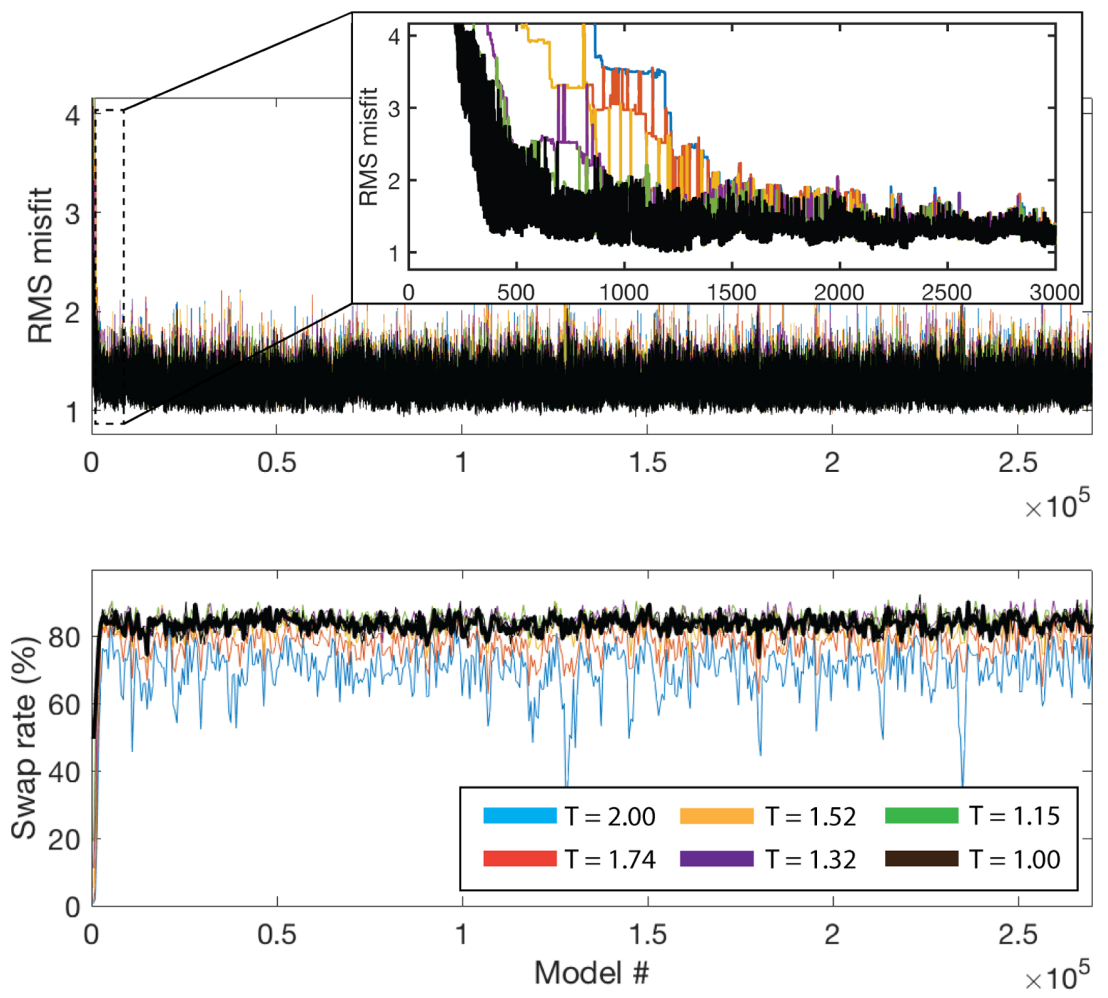

Figure 3 Convergence of Markov chains to high probability (low RMS misfit) region of model space (upper panel), and swap rate among chains (lower panel). Eight PT chains were run, three at $T=1$ (plotted in black). The chains converge rapidly from high misfit, then densely sample the low misfit region. Inset plot shows convergence to low misfit within the first several thousand steps. The swap rate indicates the temperature ladder is encouraging mixing among the eight chains. 
(a)

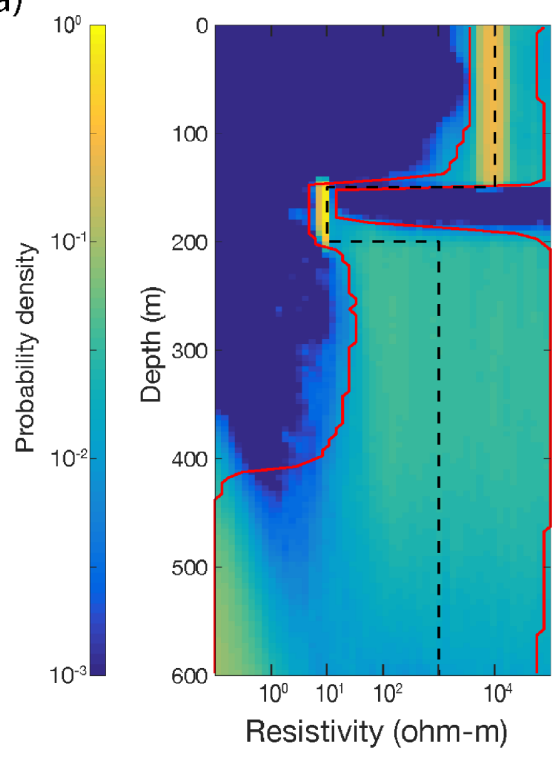

(b)

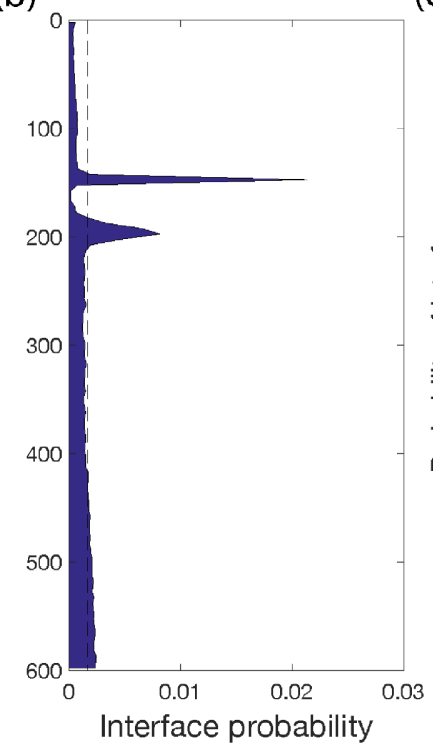

(c)

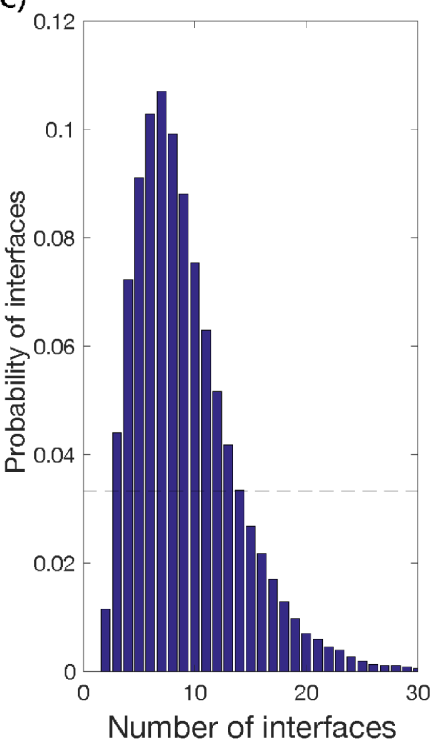

Figure 4 Inversion results from a synthetic test. The posterior probability density of resistivity (a) is plotted as a function of depth. Warmer colours indicate higher probability density. The red lines indicate the 5th and 95th percentiles at each depth-the range of resistivity between them represents the 90 per cent credible interval. The black dashed line is the true synthetic subglacial-conductor model. The probability density of interfaces as a function of depth (b), regardless of resistivity contrast, plotted against prior assumptions: a uniform distribution (dashed line). A loss of model resolution is evident below $400 \mathrm{~m}$ in both the resistivity plot, where the 90 per cent credible interval expands to encompass nearly the entire prior range, and the interface probability density plot, where the probability of an interface becomes uniform. Bayes' rule is parsimonious, leading the trans-dimensional MCMC algorithm to prefer simpler models to more complex ones, if both fit the data adequately (c). This is demonstrated in (c), where the difference between the uniform prior on $k$ (the dashed line) and the posterior on $k$ clearly indicates a preference for smaller $k$.

Fig. 4(b) is the probability density of interface location as a function of depth. The dashed line indicates the prior (a uniform distribution of interfaces).

There are a few points that can be taken from these synthetic results. First, our MCMC algorithm captures the true model in the sense that it lies within the 90 percent credible interval at nearly all depths. The second, however, is that it does more than simply recover the true model; it provides uncertainty estimates for resistivity at each depth - estimates that depend on the data, the forward modelling, and the model parametrization. For instance, Fig. 4 indicates that the airborne TEM method can much more accurately resolve conductive structures than resistive ones, as indicated by the much tighter 90 per cent credible interval over the depth range 150$200 \mathrm{~m}$. This has been known for a long time, but the Bayesian inverse method quantifies the model uncertainty. Additionally, imaging beneath a conductive structure is quite difficult for the TEM method, as the currents induced in the conductive region rapidly attenuate the primary magnetic signal and thus greatly reduce signal strength at greater depth.

A third result, demonstrated by Fig. 4(b), is that the top of a conductive layer is more sharply resolved than the bottom. This is likely the result of at least three factors - the tendency, just mentioned, of conductive bodies to mask the structures that lie beneath them; the diminishing resolution with depth; and the well-known trade-off between the thickness of a layer and its conductivity. For a buried conductive layer, the TEM method is generally more sensitive to conductance (the product of conductivity and thickness) than the layer's conductivity or thickness alone. This is because thinner, more conductive layers can produce the same TEM signal as thicker, less conductive layers. In general, the top of a conductive layer is better resolved than its base. This is evident in Fig. 4(b), where the peak at $150 \mathrm{~m}$ depth is narrower than the shorter, broader one at $200 \mathrm{~m}$.

Finally, the posterior distribution suggests that these synthetic TEM data were unable to clearly resolve the resistivity of the ice or the resistive layer beneath the conductive sediments. The location of the 95th percentile near or at the resistive boundary of the prior indicates that the upper limit on resistivity at these depths is unknown. The 5th percentile, on the other hand, indicates that both regions have a lower limit to the resistivity that is compatible with the data.

\section{APPLICATION TO FIELD DATA}

Given the success of the synthetic test, we applied our algorithm to a portion of a TEM data set collected over the McMurdo Dry Valleys (MDV) in Antarctica. The Dry Valleys are remarkable for being Antarctica's largest ice-free zone and have been extensively studied - most recently as part of a US long-term ecological research program. However, knowledge of the subsurface hydrology remains incomplete. A drilling program combined with limited seismic and DC resistivity soundings in Taylor Valley, one of the Dry Valleys, produced an unclear picture as to whether subsurface water was extensive throughout the MDV or localized (Cartwright \& Harris 1981; McGinnis \& Jensen 1971).

An extensive airborne TEM data set covering most of Taylor Valley and other areas of the MDV was collected in 2011 using the SkyTEM system (Sørensen \& Auken 2004). The data was inverted using a quasi-3-D, gradient-based inversion (Viezzoli et al. 2008) with regularization. In this framework, all of the soundings were inverted together for 3-D models with first difference smoothing in 
the lateral and vertical directions, but all forward modelling was carried out using a 1-D model beneath each sounding location. Using this inversion method, Foley et al. (2015) found an extensive, conductive feature beneath Taylor Glacier and much of Taylor Valley. They interpret this result as evidence for a layer of brine-saturated sediments that could potentially harbour microbial life (Mikucki et al. 2015).

In order to provide quantitative uncertainty bounds on the electrical resistivity beneath Taylor Valley, we applied our MCMC algorithm to two soundings over Taylor Glacier-one representative of a thin section of the glacier (sounding A, near the glacier's terminus), and the other representative of a thick section (sounding B, some $4 \mathrm{~km}$ from the terminus). See (Fig. 5). Each sounding was inverted separately. The soundings themselves are plotted in Fig. 6, along with the responses of 50 randomly sampled models from the ensemble.

Fig. 7 shows the inversion results from the two soundings. Our results are indeed consistent with the smooth, constrained inversions from Foley et al. (2015), which are plotted in white and fall almost entirely within the 90 per cent credible zone. The additional information provided by the full posterior PDF, however, is immediately clear. For instance, the upper $125 \mathrm{~m}$ and $250 \mathrm{~m}$ of the top and bottom rows, respectively, of Fig. 7 clearly indicate that the TEM data collected over Taylor Glacier are unable to resolve resistivities above $1000 \mathrm{ohm} \mathrm{m}$. Above this resistivity, over the specified depth range, the left panels of both figures look uniform - indicating that the data contain no information other than that the model must be more resistive than $1000 \mathrm{ohm} \mathrm{m}$.

Some of the lessons learned from the synthetic test are notable here as well, applied to real field data. The top middle panel of Fig. 7 clearly indicates that the top of the conductive brine channel is more sharply resolved than the bottom. Additionally, the resolution of model parameters beneath the channel is poor, and rapidly deteriorates to zero, indicated by the 90 per cent credible zone expanding to fill the entire range defined by the prior distribution. In addition, the low resistivity equivalence (conductivity-thickness trade-off) is clearly evident in the top left panel of Fig. 7: the wedge-shaped conductive zone between roughly 150 and $250 \mathrm{~m}$ depth shows that thinner conductive layers are compensated by higher conductivity, while thicker layers are offset by lower conductivity.

Fig. 7 also reveals the complex, nonlinear relationship between the depth resolution of airborne TEM data and conductivity structure. The depth sensitivity estimates of Foley et al. (2015) (indicated by the black dashed lines in the left panels of Fig. 7) are much shallower for the sounding over thin ice. This is because of the tendency of conductive layers to mask structures beneath them. Because the conductive layer is shallower in the top left panel of Fig. 7 than in the bottom left panel, the estimates of the limit of depth resolution are correspondingly shallower.

The posterior distribution obtained for the sounding over thicker ice (sounding B, Fig. 7b) merits further discussion. The bimodal distribution at $300 \mathrm{~m}$ depth seems to indicate that either regime is likely, possibly because of the inability of the data to determine the precise depth of the transition from glacier ice (resistive) to wet sediments (conductive). However, upon further inspection, the conductive mode at $300 \mathrm{~m}$ depth is found to occur only in models that also possess highly conductive layers below $350 \mathrm{~m}(<1 \mathrm{ohm} \mathrm{m})$. Because there is no likely geologic explanation for resistivity this low in sub-glacial sediments (they are more consistent with a highly saline lake, such as West Lake Bonney), we interpret these models to be among those that are mathematically acceptable (they fit the data) yet geologically unreasonable. The low probability region at $400 \mathrm{~m}$ depth (between $1-10 \mathrm{ohm} \mathrm{m}$ ) is likely a result of the competition between the geologically reasonable and unreasonable models.

One strength of Bayesian inversion is that, once the model ensemble is obtained, it can be queried for information at relatively minimal computational cost. In this case, geologically unreasonable models could be identified using some criterion and excluded when estimating the posterior. This could have the desirable effect of tightening the bounds of the posterior distribution further.

In Fig. 8, we show an example where we selected the subset of models for sounding B that have resistivity greater than $500 \mathrm{ohm}$ $\mathrm{m}$ in the upper $350 \mathrm{~m}$, and thus are compatible with the radardetermined ice thickness (Hubbard et al. 2004). By ruling out models that have conductive layers within the depth range of the glacier, we now see a significantly improved resolution of the lower limit on the resistivity of the subglacial sediments and deeper structure. Whereas the previous result had the lower limit unbounded beneath $350 \mathrm{~m}$, the new result suggests resistivity beneath the glacier is at least a few ohm $\mathrm{m}$. In the remaining sections the full, unconstrained model ensemble was used in order to demonstrate the fundamental resolution of airborne TEM data. Improved resolution could be obtained, however, if independent constraints - such as ice thickness - are incorporated, as demonstrated in Fig. 8.

\subsection{Model resolution and depth of investigation}

A comparison of the top and bottom rows of Fig. 7 reveals the effect of increasing depth on the resolving power of TEM data. That resolution should decrease with depth is not surprising, given the diffusive nature of the TEM method. But it prompts the important question of how deep one can resolve electrical conductivity using airborne TEM. Often referred to as the DOI, this question seeks the depth below which the data are no longer sensitive to changes in the model. A first-order method for depth sensitivity estimation is the skin-depth, which is the depth at which the primary signal has been attenuated to $1 / e$ of its original strength. However, the skin-depth assumes a conductive whole space and does not take into account the nonlinear effects of conductivity structure or the uncertainty of the data.

A more sophisticated approach that is in common use is to compute a Jacobian sensitivity matrix (the matrix of partial first derivatives of the data with respect to the model parameters) and use the norm of each column of the matrix as a measure of the sensitivity of the data to the corresponding model parameter (Christiansen \& Auken 2012). While the sensitivity of airborne TEM data clearly decays in a continuous fashion (rather than abruptly, at the DOI), the point at which the sensitivity measure falls below a certain threshold can be used as a rough guide to where the model can no longer be trusted.

This method of measuring DOI is a good choice for inverse methods that produce only one best-fit model. Its weakness, however, is that it is a linearization about only one model. It is conceivable that this DOI measure could be substantially different for other models that fit the data equally well. Additionally, if the data dependence on the model parameters is highly nonlinear about the chosen model (e.g. models with complicated structure or large trade-offs), this DOI estimate may not be accurate.

Having access to the full posterior PDF allows a more complete answer to the question: how much can I trust my model as a function of depth? The complete answer is contained in the posterior- that is, the shape of the posterior will indicate precisely how well the data are able to resolve each portion of the model. Areas where the 


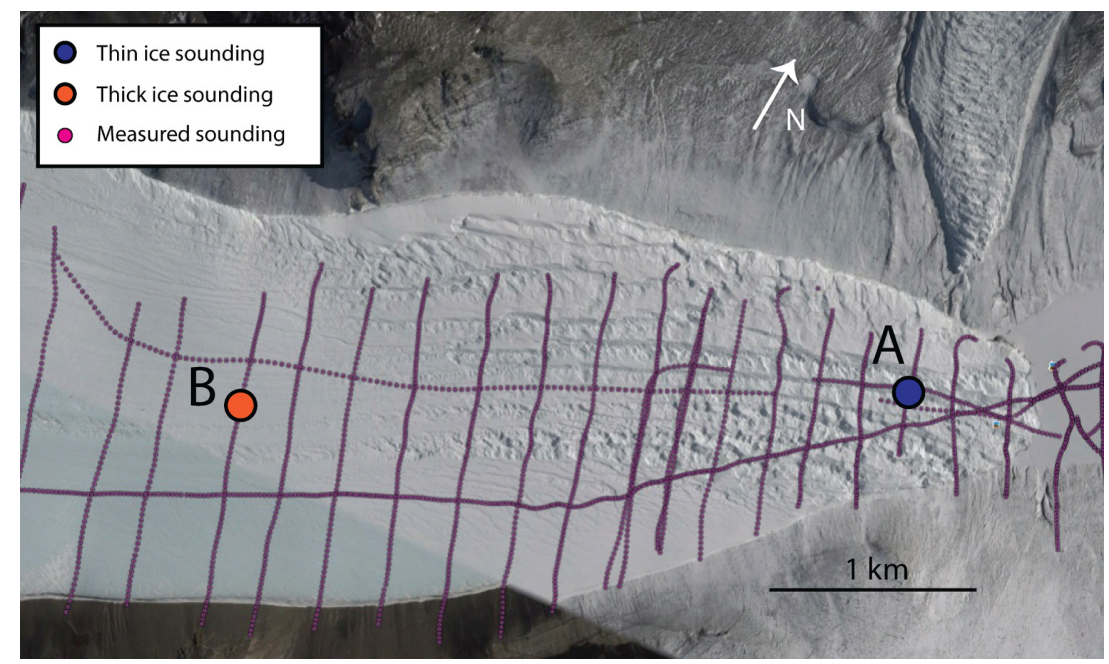

Figure 5 Map of Taylor Glacier, at the head of Taylor Valley, Antarctica. Soundings measured during the survey are marked in pink. Soundings we inverted using our MCMC algorithm in this study are marked in blue (A, over relatively thin ice) and orange (B, over relatively thicker ice).
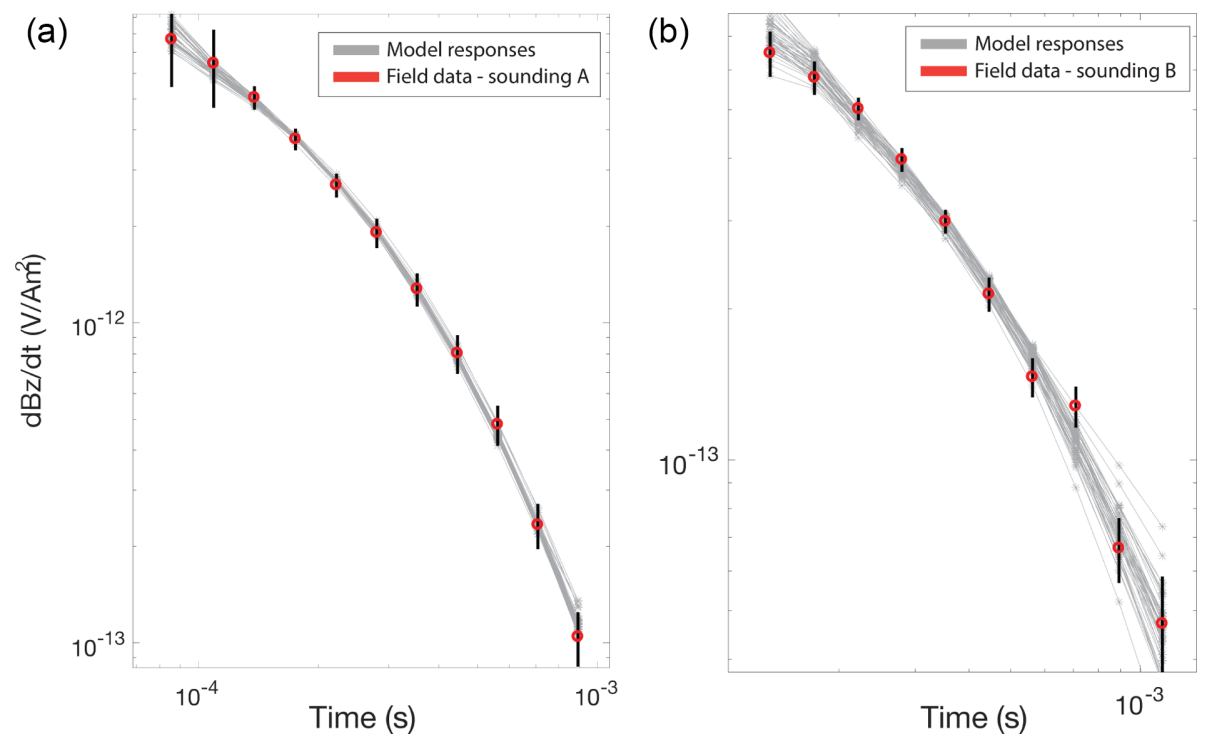

Figure 6 Field data, plotted as red circles, from soundings A (left) and B (right). The responses of 50 models chosen at random from the model ensemble are plotted in grey.

data provide minimal illumination will closely resemble the prior distribution and our prior assumptions about the model will remain unchanged. On the other hand, areas where the data illuminate the model should reveal marked differences from the prior. For example, the bottom left panel of Fig. 7 below $500 \mathrm{~m}$ looks scarcely different from a uniform distribution (our prior assumption), indicating that the data are incapable of resolving the model parameters at these depths. By contrast, the top left panel of Fig. 7 at a depth of about $175 \mathrm{~m}$ is focused and, by any measure, very dissimilar to a uniform distribution. In other cases, the posterior may look quite different from the prior and yet be poorly resolved - for example, the bottom left panel of Fig. 7 at a depth of $300 \mathrm{~m}$, which is bimodal.

While the most complete picture is the posterior itself, it is occasionally useful to summarize the information it contains more concisely. One such concise estimate of the DOI derived from the posterior is the Kullback-Leibler (KL) divergence, which is a measure of the relative entropy of two distributions.
Entropy is a measure of randomness and can be used to quantify the information content of a distribution. For a discrete distribution, it is defined as (MacKay 2003)

$H(P)=-\sum_{i} P\left(x_{i}\right) \log P\left(x_{i}\right)$

where the above is defined at $P\left(x_{i}\right)=0$ since $\lim _{P \rightarrow 0+} P \log (P)=0$.

To understand the connection with information, consider the case of a discrete uniform distribution with probability $U_{N}=\frac{1}{N}$. The entropy of this distribution is

$$
\begin{aligned}
H\left(U_{N}\right) & =-\sum_{i=1}^{N} \frac{1}{N} \log \frac{1}{N} \\
& =\log N .
\end{aligned}
$$

The larger $N$, the wider $U_{N}$ 's bounds and the less information it contains - or, in other words, the larger its entropy, which grows per 



Figure 7 Inverted results for a sounding near the terminus of Taylor Glacier (a), and a sounding $4 \mathrm{~km}$ further up the glacier (b). For a description of each panel, see Fig. 4. The black dashed lines in the left panels are two linearized estimates of the limit of the data's depth sensitivity to the model (Christiansen \& Auken 2012). The white dashed lines indicate our estimate of the ice thickness, using the posterior. The solid white lines show the regularized inversion result of Foley et al. (2015) at the respective locations. The black line is the median of the distribution at each depth. The wedge-shaped region between 150 and $250 \mathrm{~m}$ depth in the resistivity distribution of the thin ice sounding (a) reveals the trade-off between conductivity and layer thickness. In the thin ice sounding, the upper boundary of the conductive feature is more sharply resolved than the bottom. The depth sensitivity estimates for the thick ice sounding are much deeper than for the thin ice sounding, indicating that depth sensitivity of TEM data is a complex, nonlinear function of conductivity structure.

Equation 8 as the $\log$ of $N$. The KL divergence measures the relative entropy of two distributions and is defined, for discrete distributions, as (MacKay 2003)

$D_{\mathrm{KL}}(P \| Q)=\sum_{i} P\left(x_{i}\right) \log \frac{P\left(x_{i}\right)}{Q\left(x_{i}\right)}$.
In a Bayesian context, $Q$ and $P$ in eq. (9) represent the prior and posterior, respectively. The posterior differs from the prior due to the additional information contained in the data. The KL divergence measures this information gain. To see how this is so, consider again 

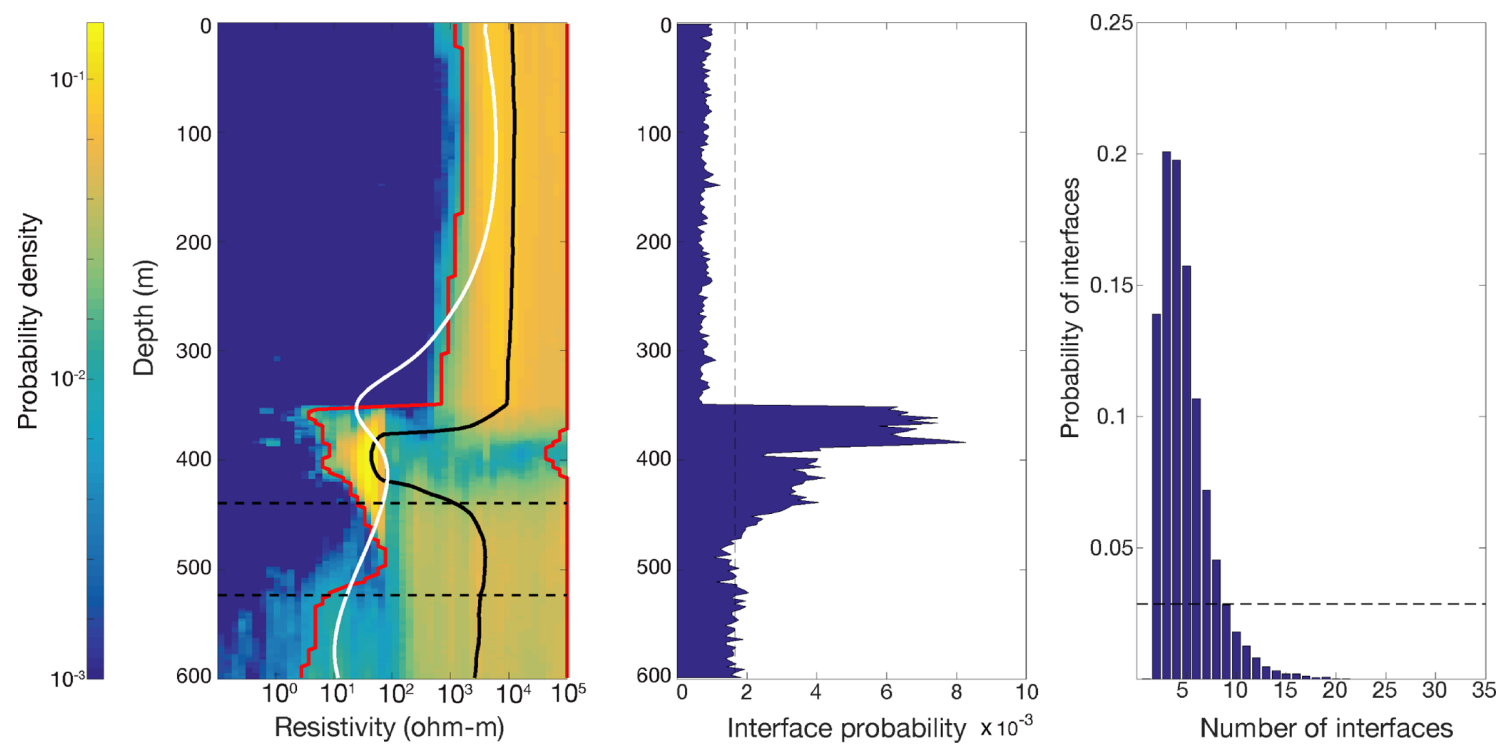

Figure 8 Inverted results for sounding B using only those models from the full ensemble whose resistivity was compatible with that of glacial ice ( $>500$ $\mathrm{ohm} \mathrm{m}$ ) above $350 \mathrm{~m}$, the depth to the glacier bottom (determined from radar soundings). Compare with Fig. 7(b). Note the lower limit, indicated by the 5th percentile, on the electrical resistivity in the subglacial sediments, which was unbounded when the full model ensemble was used to estimate the posterior. Also note that the conductivity in the region of the wet, conductive sediments now has the same wedge-like shape as Fig. 7(a).

a uniform prior, $Q=U_{N}$. The KL divergence of $P$ and $Q$ is then

$$
\begin{aligned}
D_{\mathrm{KL}}(P \| Q) & =\sum_{i=1}^{N} P\left(x_{i}\right) \log \frac{P\left(x_{i}\right)}{Q\left(x_{i}\right)} \\
& =\sum_{i=1}^{N} P\left(x_{i}\right) \log P\left(x_{i}\right)-\sum_{i=1}^{N} P\left(x_{i}\right) \log Q\left(x_{i}\right) \\
& =\sum_{i=1}^{N} P\left(x_{i}\right) \log P\left(x_{i}\right)-\log \frac{1}{N} \sum_{i=1}^{N} P\left(x_{i}\right) \\
& =-H(P)-\log \frac{1}{N} \\
& =H\left(U_{N}\right)-H(P) .
\end{aligned}
$$

By rewriting eq. (9) in terms of entropy, the connection to information gain is apparent: in a Bayesian context, the KL divergence represents the entropy loss (or conversely, the information gain) in going from the uniform prior to the posterior. Put another way, it measures the information the data have added to our prior knowledge.

We computed the KL divergence at each depth for the two soundings of Fig. 7 to investigate their depth sensitivity. Fig. 9 shows the KL divergence as a function of depth for these two soundings, plotted next to their resistivity PDFs. Over the depth range of the conductive sediments, the KL divergence of the sounding over thin ice shows a strong increase against a background of decreasing resistivity with depth. The KL divergence of the sounding over thick ice shows the same drop off with depth, but the signal indicating the presence of the conductive layer, while noticeable, is much weaker since it occurs $200 \mathrm{~m}$ deeper.

\subsection{Mining the ensemble for information}

The Bayesian method is highly versatile: once the model ensemble has been obtained, extracting information from it becomes a matter of data mining. For instance, the sensitivity of the airborne TEM method to conductance can be quantitatively measured from the ensemble. This is feasible mostly for a buried conductor in a relatively uniform resistive background. For each sounding, we selected a depth range over which the posterior indicated low resistivity consistent with the presence of a conductive channel (more conductive than expected for background rock or overlying ice). For sounding A, the depth range used was 140-225 m; for sounding $\mathrm{B}, 345-445 \mathrm{~m}$. We then calculated the conductance of each model in the ensemble by integrating conductivity over that depth range. Histograms of conductance, computed as described, for the two soundings are shown in Fig. 10. While this is a somewhat subjective process, we found that adjusting the intervals slightly had only a minor effect on the conductance histograms.

Most of the signal in the histogram for the thin ice sounding is concentrated in a peak around about $1.8 \mathrm{~S}$ (Siemens) that spans only $2 \mathrm{~S}$ in width, while the upper left panel of Fig. 7 shows that resistivity over the same depth range spans roughly two orders of magnitude. Meanwhile, the sounding over the thick ice indicates a similar peak in conductance at about $2 \mathrm{~S}$, but reveals much greater uncertainty due to the conductive layer's greater depth. The usefulness of quantifying conductance is especially apparent when comparing Fig. 10 with the resistivity PDF of the sounding over thick ice (lower left panel of Fig. 7). The 90 per cent credible interval spans nearly 5 orders of magnitude of resistivity, yet the conductance histogram for this sounding yields a clear peak at the same value as the sounding over thin ice, suggesting that, despite the diminished resolution, the data are detecting the same conductive channel in both soundings.

In the course of interpreting inversion results, one is often interested in variables other than, but related to, those estimated in the model. For instance, a scientist interpreting inverted models of electrical conductivity might be interested, depending on the survey region, in deriving pore fluid salinity, the porosity of sediments, partial melt fraction, or estimating net hydrocarbons. Using standard inversion techniques, an interpreter is obligated to rely on a single model estimate, without associated uncertainties, to infer the value of the desired property. Access to the full posterior and a function relating the model variable to the desired quantity make it possible 

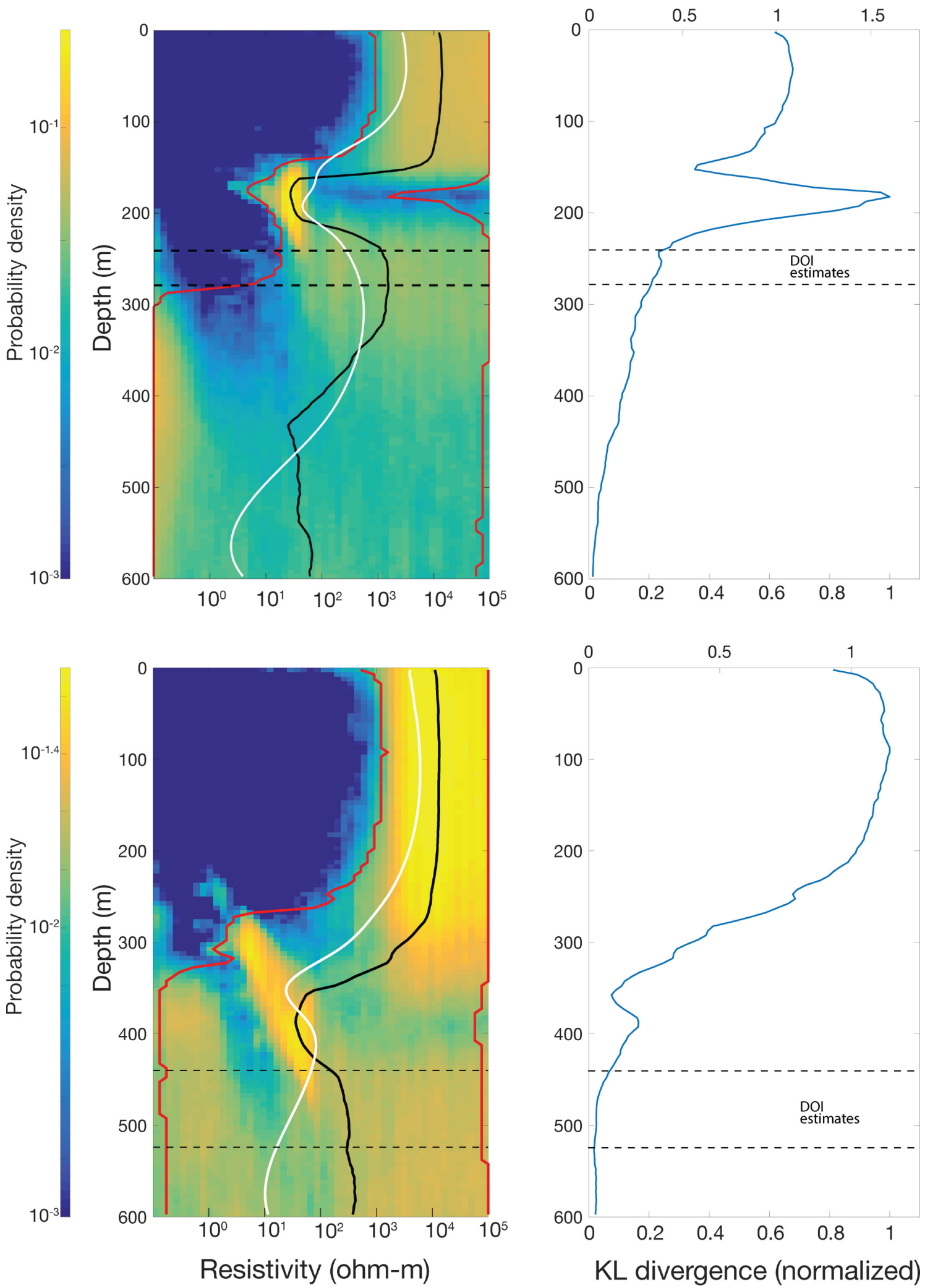

Figure 9 Resistivity PDFs (left column) and Kullback-Leibler (KL) divergences (right column) for a sounding over thin ice (top row) and thick ice (bottom row). The axis along the top of plots in the column on the right is the unnormalized KL divergence, while the axis along the bottom of each plot is the KL divergence, normalized to the maximum for that particular sounding. The large peak in KL divergence in the upper right panel against a steady decline with depth indicates that the data have clearly resolved the conductive channel. By contrast, the small peak at $400 \mathrm{~m}$ depth in the lower right panel suggests that the conductive channel is much less well resolved in the sounding over thick ice. 


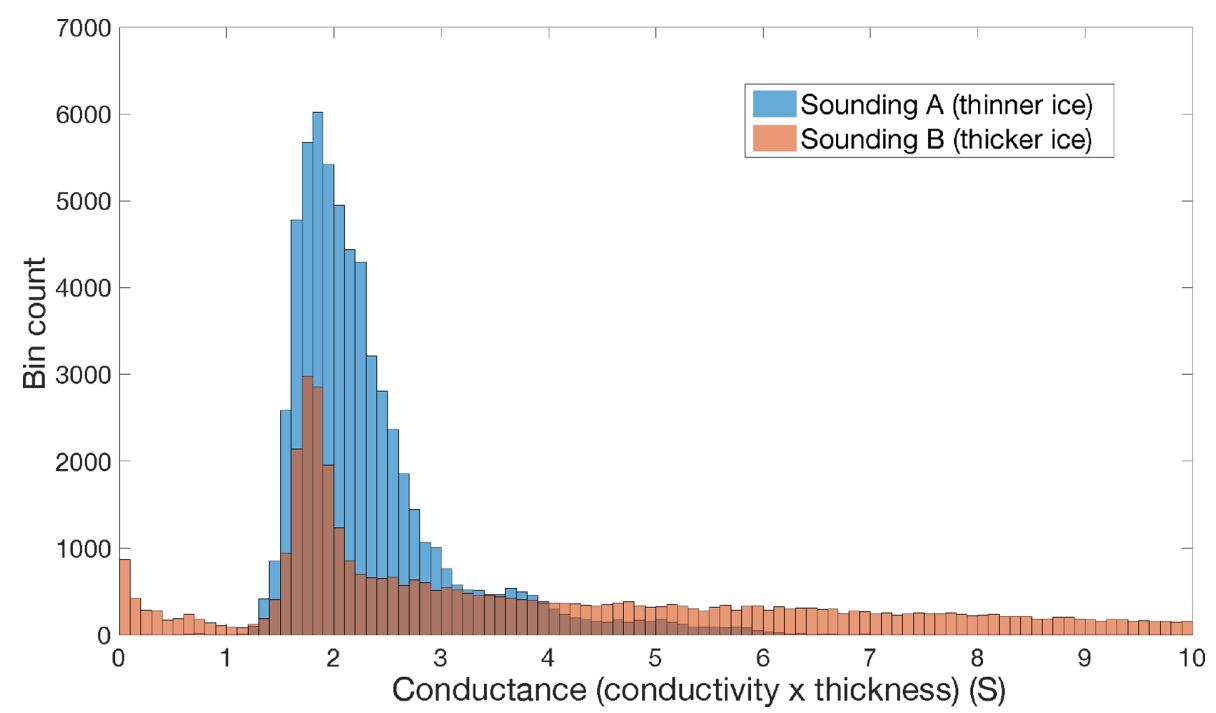

Figure 10 Conductance histograms for the thin ice (blue) and thick ice (orange) soundings. The distribution of the thin ice sounding is concentrated over a range of only $2 \mathrm{~S}$, whereas its resistivity is distributed over nearly two orders of magnitude (see Fig. 7). Despite the much greater spread due to diminished data sensitivity at depth, the thick ice sounding features a sharp peak at the same value as the thin ice sounding.

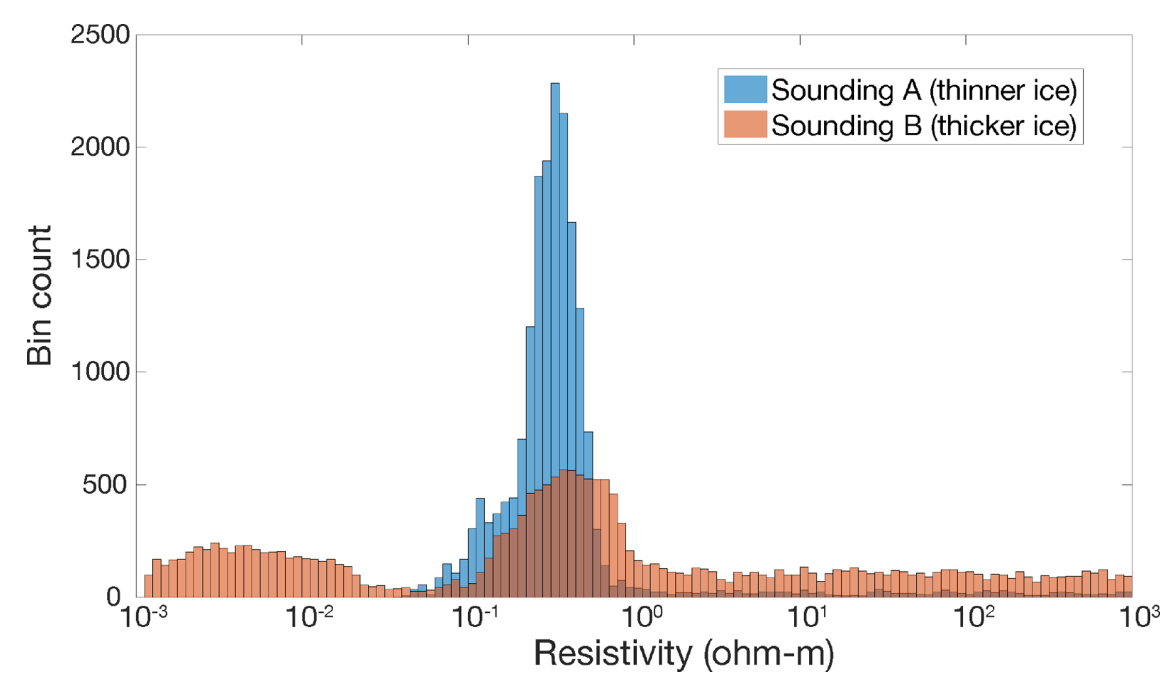

Figure 11 Histograms of pore fluid resistivity, $\rho_{w}$, in the conductive sediments beneath Taylor Glacier obtained from Archie's Law assuming $\phi=10$ per cent and $m=2$. The bulk resistivity values, $\rho$, were obtained from the model ensemble. While the sounding over thin ice is sharply peaked, the sounding over thick ice yields a broader distribution, indicating decreasing resolution with depth. Despite this, the mode of both distributions is nearly identical.

to produce an entire estimated PDF of these derived quantities of interest, rather than just one plausible value.

To illustrate the utility of Bayesian inversion in estimating related parameters, we used Archie's Law (Archie 1942)

$\rho=\rho_{w} \phi^{-m}$,

where $\rho$ is electrical resistivity, $\rho_{w}$ is pore fluid resistivity, $\phi$ is porosity, and $m$ is an empirically derived constant with a typical value of 2 . For a given sounding, we selected a depth, $z_{\text {brine }}$, consistent with the centre of the conductive channel, using both the resistivity PDF and interface PDF as guides. In particular, we chose the depth of $z_{\text {brine }}$ to capture the widest possible variation in resistivity. In practice, this meant choosing $z_{\text {brine }}$ to correspond with the thickest portion of the conductivity-thickness trade-off wedge. For sounding A, this was chosen to be $175 \mathrm{~m}$; for sounding B, $400 \mathrm{~m}$. Then, for each model in the ensemble, we compute the pore fluid resistivity, using Archie's Law, assuming a porosity of 10 per cent and $m=2$, and the bulk resistivity of the model at $z_{\text {brine }}$. We then produced a histogram of pore fluid resistivity at that depth, using the values computed from the model ensemble.

We followed this procedure for the thin ice and thick ice soundings. The results, shown in Fig. 11, quantitatively show the diminishing resolution with increased ice thickness (i.e. depth to the conductor). For example, the inter-quartile range ( 50 per cent credible interval) for the thin ice sounding gives a likely range of 0.25 to $0.42 \mathrm{ohm} \mathrm{m}$, with a median value of $0.32 \mathrm{ohm} \mathrm{m}$. The thick ice sounding, by contrast, yields a likely range of 0.03 to $5.0 \mathrm{ohm} \mathrm{m}$, with a median value of $0.43 \mathrm{ohm} \mathrm{m}$, reflecting the much greater uncertainty inherent in trying to image a deep target. Despite the greater uncertainty, the median value is nearly the same between the two soundings, potentially giving rise to a false sense of certainty about the value of pore fluid resistivity from the thick ice sounding if only one estimate were available. The uncertainty information contained in these histograms might be of use to, say, a 

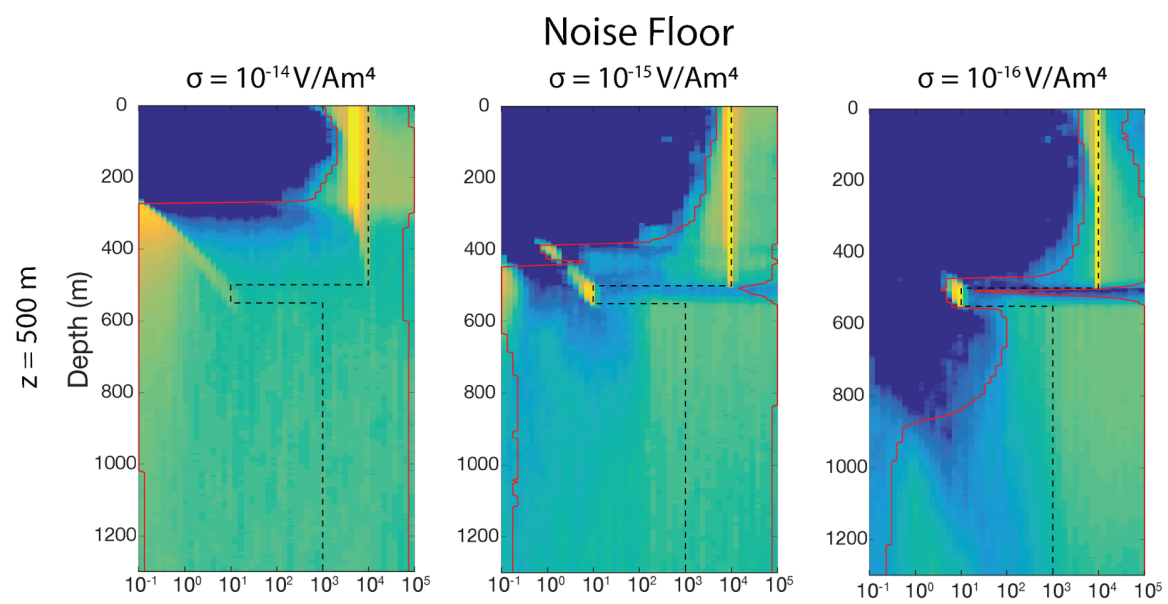

\section{$\mathrm{KL}$ divergence}
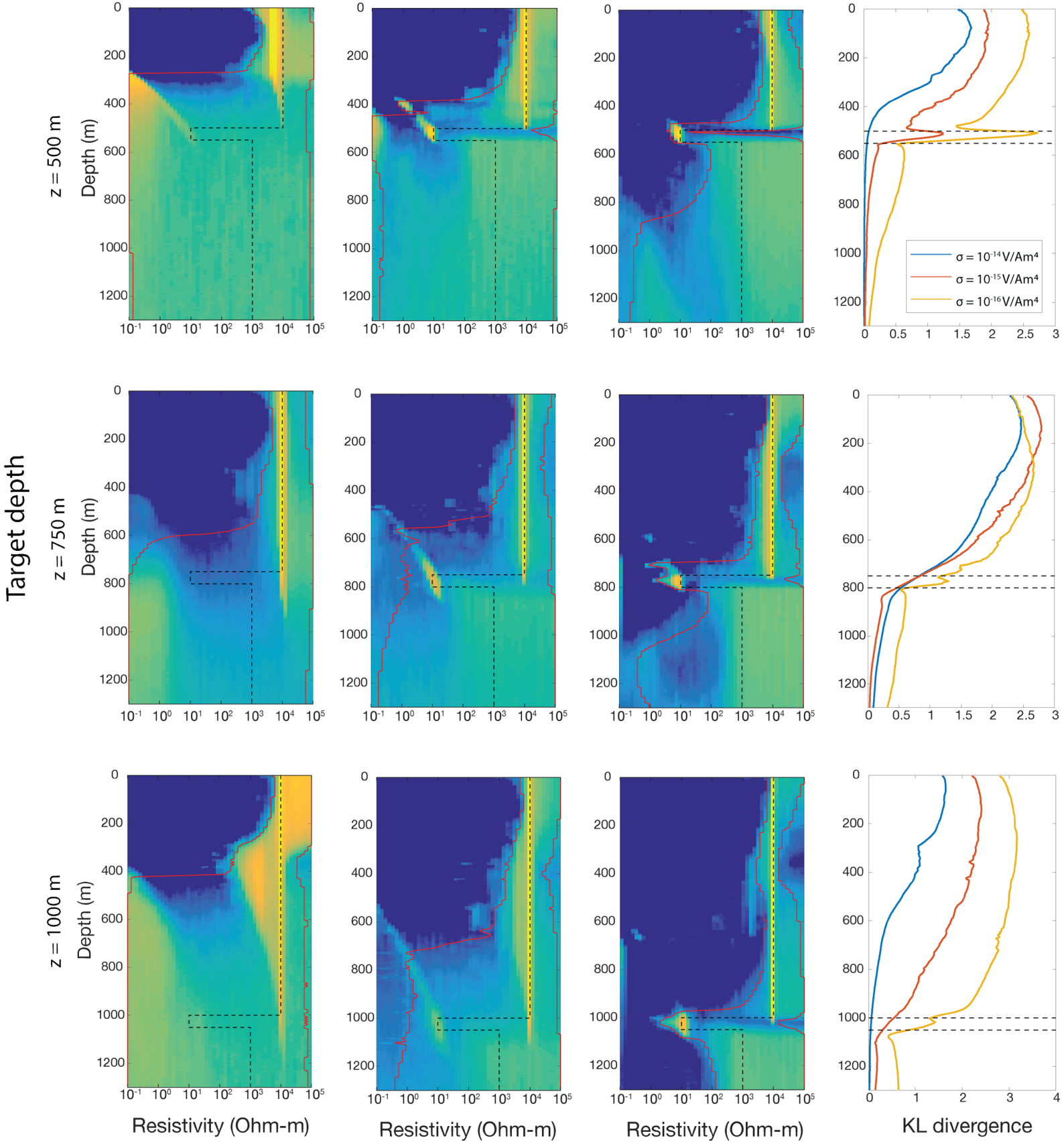

Figure 12 Depth test (i.e. how deep can TEM see?). Columns represent $10^{-14} \mathrm{~V} \mathrm{Am}^{-4}$ (left), $10^{-15} \mathrm{~V} \mathrm{Am}^{-4}$ (centre-left), $10^{-16} \mathrm{~V}$ Am ${ }^{-4}$ (centre-right) and the KL divergence for each noise floor (right). Rows represent depth: $z=500 \mathrm{~m}$ (top), $z=750 \mathrm{~m}$ (centre), $z=1000 \mathrm{~m}$ (bottom).

microbiologist seeking to understand and constrain the range of environmental conditions that could exist within the brine layer. Given the assumed 10 per cent porosity, the median value of pore fluid resistivity is consistent with that of seawater at $1{ }^{\circ} \mathrm{C}(0.3 \mathrm{ohm}$ $\mathrm{m})$. Taylor Valley was once covered by oceans, suggesting that the fluid filling the pore space of the sediments beneath the valley today might be ancient seawater.

\section{FEASIBILITY OF DEEPER IMAGING}

As evidenced by Fig. 9, the ability of the data to resolve the conductive channel beneath Taylor Glacier diminishes as the thickness of the glacier increases. Because measurement error is a source of model parameter uncertainty, it might be possible to increase model resolution at depth by decreasing absolute measurement error, which would allow for data at later time offsets that provide increased sensitivity to deeper structure. In the context of TEM, this could be accomplished by placing the transmitter loop directly on the ice in lieu of towing it under a helicopter. This sacrifices data acquisition efficiency, but allows for repeat soundings to be collected at the same location which can subsequently be stacked to reduce the effect of measurement error. Of course, as the depth (and lateral) sensitivity of the data increase, the 1-D assumption will eventually 

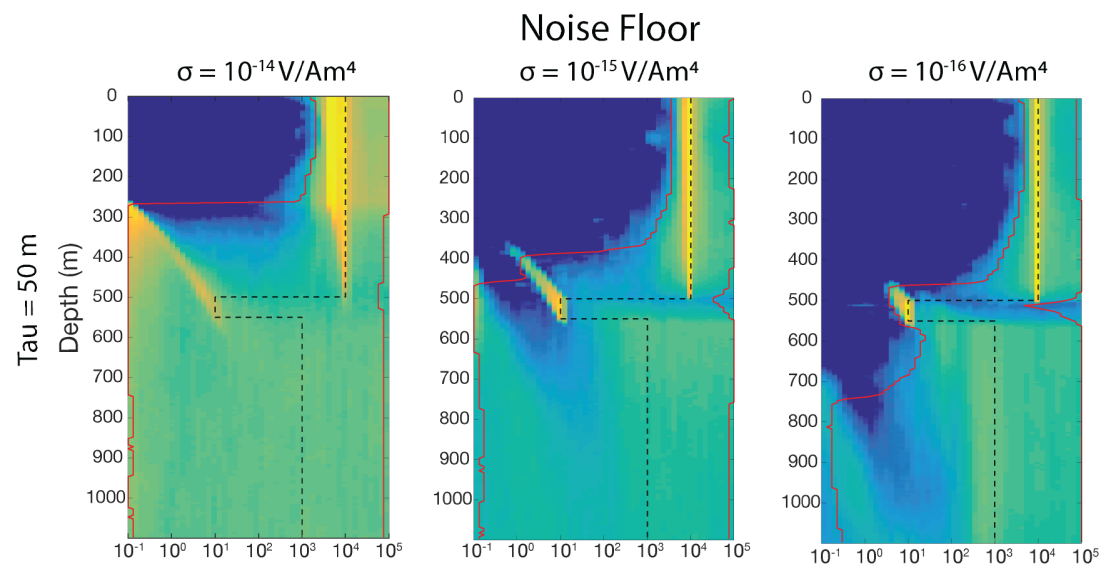

\section{$\mathrm{KL}$ divergence}
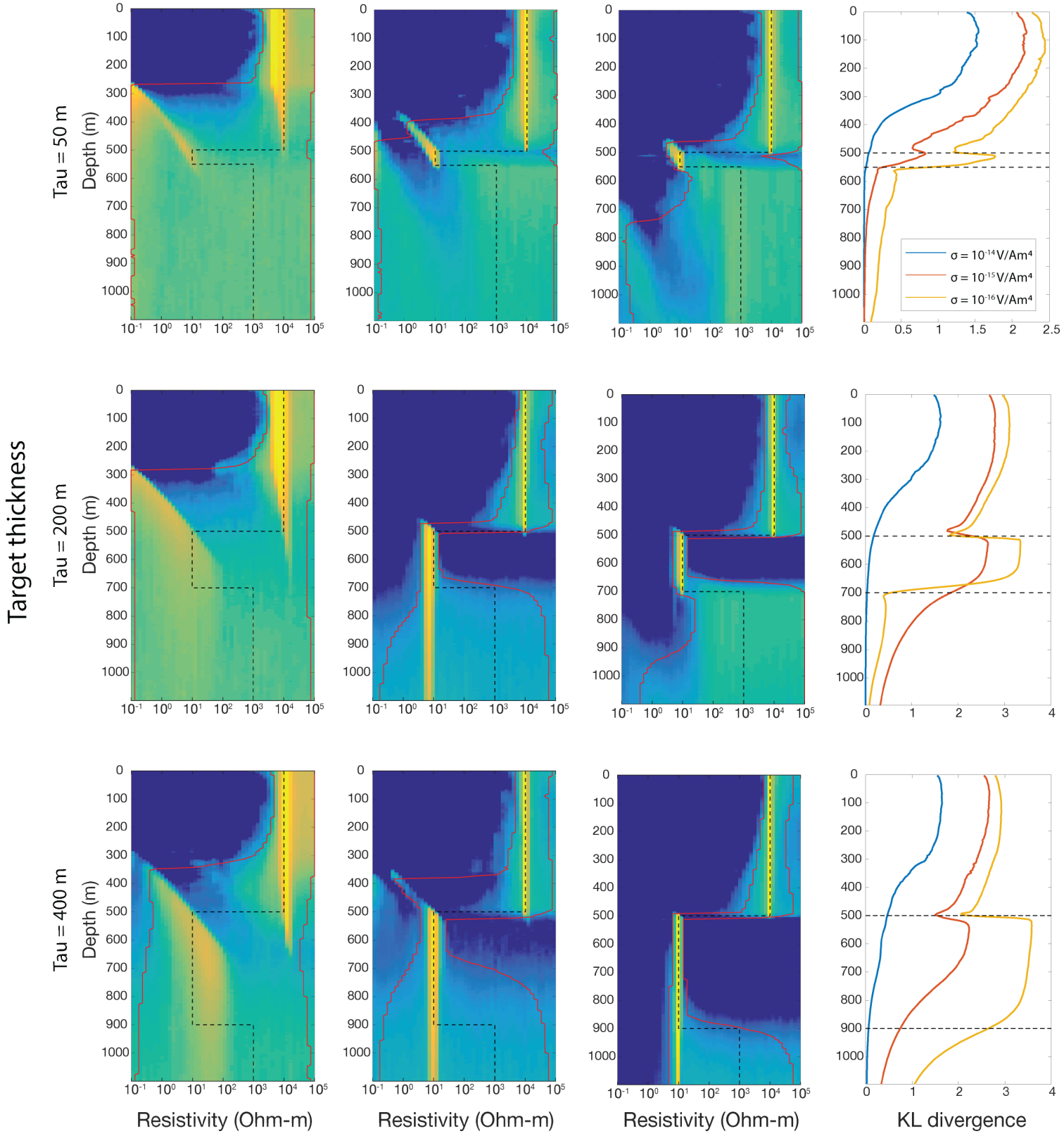

Figure 13 Thickness test (can we see the bottom?). Columns represent $N=1$ (left), $N=100$ (centre-left), $N=10000$ (centre-right) and the KL divergence for each $N$ value (right). Rows represent thickness: $\tau=50 \mathrm{~m}$ (top), $\tau=200 \mathrm{~m}$ (centre), $\tau=400 \mathrm{~m}$ (bottom)..

break down. Because they are fundamentally stochastic, Bayesian sampling methods should be able to quantify this improvement in resolution capacity, providing valuable insight into trade-offs between efficiency and resolution that are inherent in experimental survey design.

To determine what impact a decrease in measurement error would have on the ability to resolve the conductive channel under greater ice thicknesses, we conducted a synthetic experiment where the same conductive layer ( $50 \mathrm{~m}$ thick, $10 \mathrm{ohm} \mathrm{m}$ resistivity) was buried under increasingly thick layers of ice. Additionally, for each depth of ice $(500,750$ and $1000 \mathrm{~m})$, we decreased the noise floor by an order of magnitude to simulate the power of a larger transmitter moment (e.g. $75 \mathrm{~A}$ in a $100 \times 100 \mathrm{~m}$ loop), longer data stacking times, or both. As before, the noise in the synthetic data consisted of a 5 per cent relative Gaussian error, plus an absolute Gaussian noise centred at the noise floor, which we lowered from $10^{-14} \mathrm{~V} \mathrm{Am}^{-4}$ (consistent with the airborne TEM data collected in 2011) to $10^{-16}$ $\mathrm{V} \mathrm{Am}^{-4}$. Synthetic data below the noise floor were discarded.

One additional difference is that the synthetic data with a noise floor of $10^{-14} \mathrm{~V} \mathrm{Am}^{-4}$ were modelled assuming a transmitter height of $35 \mathrm{~m}$, typical of airborne TEM surveys, while the synthetic data with lower noise floors were modelled assuming the transmitter was located on the surface. The intent was to allow comparison of airborne TEM to ground based TEM data.

We then inverted each synthetic data set. The results are shown in Fig. 12. Each row reveals how the decreasing the noise floor increases the resolving power of the data. Note how a noise floor of $10^{-14} \mathrm{~V} \mathrm{Am}^{-4}$ is entirely insufficient to resolve the conductive 
layer buried at any of the depths considered. A noise floor of $10^{-15}$ $\mathrm{V} \mathrm{Am}^{-4}$, by contrast, is sufficient to partially resolve the layer, but the data still permit many other models to satisfy the data, complicating interpretation. A noise floor of $10^{-16} \mathrm{~V} \mathrm{Am}^{-4}$, however, permits an unambiguous interpretation at all three depths, with a distribution clearly focused and centred on the true model. The KL divergence indicates that the data contain increasing amounts of information about the conductive layer as the noise floor decreases. The highest noise floor registers nothing other than a steady decline in information content with depth for all target depths. The intermediate noise floor data show a clear spike in information content regarding the target at $500 \mathrm{~m}$ depth, but not at greater depths. The lowest noise floor, by contrast, shows a clear increase in information at the appropriate depth for all burial depths shown, although the signal decreases markedly with increasing burial depth.

Another interesting question regarding the resolving power of TEM data with depth is whether the bottom of a conductive layer can be clearly resolved. Most often, the top of a conductive layer is better resolved than the bottom, both because of the conductivitythickness trade-off and because the conductive layer dissipates the EM signal more rapidly than the more resistive overburden. The bottom row of Fig. 9 effectively demonstrates the difficulty: it is possible that $450 \mathrm{~m}$ represents the bottom of the conductive layer, but the loss of resolution at that same depth-indicated by both the linearized estimates of DOI and by the KL divergence - renders interpretation uncertain.

To investigate whether increasing the information content of the data by lowering the noise floor could resolve this issue, we conducted a second synthetic test. Keeping the top of the conductive layer fixed at $500 \mathrm{~m}$ (with the same $10 \mathrm{ohm}$ m resistivity as before), we varied the layer's thickness. We generated synthetic data using the same procedure as above, dropping the noise floor by an order of magnitude each time, and inverted each using our MCMC algorithm.

The results of this synthetic experiment are shown in Fig. 13. As before, the airborne TEM setup (highest noise floor and $35 \mathrm{~m}$ transmitter height) is unable to resolve any of the three layers, regardless of thickness. If the noise floor is an order of magnitude lower and the thickness of the conductive layer is $50 \mathrm{~m}$, it is unclear whether the bottom of the layer has been identified. Because TEM data contains little information about deeply buried resistors, without knowledge of the true model it would be difficult to say whether the drop in KL divergence at $550 \mathrm{~m}$ - the 'true' bottom of the layer - is due to a loss of signal strength or to the transition from the conductive layer to a more resistive underburden. The posterior PDF suggests that these two factors are combining to cause a loss of resolution at the same depth, making it impossible to distinguish between them or say definitively that the bottom of the layer has been imaged.

By lowering the noise floor further, this ambiguity is resolved. Below $550 \mathrm{~m}$, the 5 th percentile of the distribution rises above the resistivity of the conductive layer, indicating that nearly all of the models below $550 \mathrm{~m}$ are too resistive to represent a continuation of the conductive layer. If the layer thickness is increased to $200 \mathrm{~m}$, the inverted results are again ambiguous if the noise floor is intermediate: many of the models have the conductive layer continuing indefinitely, revealing that these models fit the data just as well as the true model. If the noise floor is decreased further to $10^{-16} \mathrm{~V}$ $\mathrm{Am}^{-4}$, however, the bottom of the layer is identifiable, using the 5 th percentile of the distribution below $700 \mathrm{~m}$ - the 'true' bottom of the layer - as evidence. If the layer thickness is further increased to $400 \mathrm{~m}$, however, information about the depth of the bottom of the layer is lost, and all three synthetic data sets show ambiguity at depth.

\section{CONCLUSIONS}

We applied a trans-dimensional MCMC algorithm to provide Bayesian inference on model parameters estimated from airborne TEM data. We utilized parallel tempering to accelerate convergence and improve robustness of the algorithm, and demonstrated its efficacy on both synthetic TEM data and field data recorded over the Taylor Glacier in Antarctica. Our estimated resistivity distributions corroborate the results of previous inversions of this data set using standard, gradient-based techniques. They also provide a wealth of additional information, including quantitative estimates of model parameter uncertainty, a statistical approach to DOI estimation derived from the KL divergence, and quantitative estimation of related subsurface parameters, including conductance and pore fluid resistivity. This last point is of significance, as it is often the potential to place constraints on related subsurface parameters that is of greatest interest to the wider scientific community. The parsimonious nature of the trans-dimensional MCMC algorithm means that the data largely drive the choice of model complexity, implicitly preferring simpler models to more complex ones - rather than placing this decision in the hands of the user. The generality of the MCMC algorithm means it can be adapted to work with other types of geophysical data.

\section{ACKNOWLEDGEMENTS}

We gratefully acknowledge funding support from National Science Foundation grants 1043618 and 1344348 which funded the original data collection and interpretation.

\section{REFERENCES}

Agostinetti, N.P. \& Malinverno, A., 2010. Receiver function inversion by trans-dimensional Monte Carlo sampling, Geophys. J. Int., 181, 858-872.

Archie, G.E., 1942. The Electrical Resistivity Log as an Aid in Determining Some Reservoir Characteristics, Trans. AIME, 146(1), 54-62.

Auken, E. \& Christiansen, A.V., 2004. Layered and laterally constrained 2D inversion of resistivity data, Geophysics, 69(3), 752-761.

Brodie, R. \& Sambridge, M., 2012. Transdimensional Monte Carlo inversion of AEM data, ASEG Extended Abstracts, 2012(1), 1-4.

Cartwright, K. \& Harris, H. J.H., 1981. Hydrogeology of the dry valley region, Antarctica, in Dry Valley Drilling Project, ed Lyle, D. Mcginnis, pp. 193-214, American Geophysical Union.

Christiansen, A.V. \& Auken, E., 2012. A global measure for depth of investigation, Geophysics, 77(4), WB171-WB177.

Cox, L.H., Wilson, G.A. \& Zhdanov, M.S., 2012. 3D inversion of airborne electromagnetic data, Geophysics, 77(4), WB59-WB69.

Dettmer, J., Dosso, S.E. \& Holland, C.W., 2010. Trans-dimensional geoacoustic inversion, J. acoust. Soc. Am., 128(6), 3393-3405.

Dettmer, J., Molnar, S., Steininger, G., Dosso, S.E. \& Cassidy, J.F., 2012. Trans-dimensional inversion of microtremor array dispersion data with hierarchical autoregressive error models, Geophys. J. Int., 188(2), 719734.

Dosso, S.E., Holland, C.W. \& Sambridge, M., 2012. Parallel tempering for strongly nonlinear geoacoustic inversion, J. acoust. Soc. Am., 132(5), 3030-3040.

Dosso, S.E., Dettmer, J., Steininger, G. \& Holland, C.W., 2014. Efficient trans-dimensional Bayesian inversion for geoacoustic profile estimation, Inverse Probl., 30(11). 
Falcioni, M. \& Deem, M.W., 1999. A biased Monte Carlo scheme for zeolite structure solution, J. Chem. Phys., 110(3), 1754-1766.

Fitterman, D.V. \& Anderson, W.L., 1987. Effect of transmitter turn-off time on transient soundings, Geoexploration, 24(2), $131-146$.

Foley, N., Tulaczyk, S., Auken, E., Schamper, C., Dugan, H., Mikucki, J., Virginia, R. \& Doran, P., 2015. Helicopter-borne transient electromagnetics in high-latitude environments: An application in the McMurdo Dry Valleys, Antarctica, Geophysics, 81(1), WA87-WA99.

Fountain, D., Smith, R., Payne, T. \& Limieux, J., 2005. A helicopter timedomain EM system applied to mineral exploration: system and data, First Break, 23(11).

Galetti, E., Curtis, A., Baptie, B., Jenkins, D. \& Nicolson, H., 2016. Transdimensional Love-wave tomography of the British Isles and shear-velocity structure of the East Irish Sea Basin from ambient-noise interferometry, Geophys. J. Int., 208(1), 36-58.

Gehrmann, R.A.S., Dettmer, J., Schwalenberg, K., Engels, M., Dosso, S.E. \& Özmaral, A., 2015. Trans-dimensional Bayesian inversion of controlledsource electromagnetic data in the German North Sea, Geophys. Prospect., 63(6), 1314-1333.

Geyer, C.J., 1991. Markov chain Monte Carlo maximum likelihood, in Computing Science and Statistics: Proceedings of the 23rd Symposium on the Interface, pp. 156-163, eds Keramidas, E.M. \& Kaufman, S.M., New York.

Geyer, C.J. \& Moller, J., 1994. Simulation procedures and likelihood inference for spatial point processes, Scand. J. Stat., 21(4), 359-373.

eds Gilks, W.R., Richardson, S. \& Spiegelhalter, D., 1995. Markov Chain Monte Carlo in Practice, CRC Press.

Green, P.J., 1995. Reversible jump Markov chain Monte Carlo computation and Bayesian model determination, Biometrika, 82(4), 711-732.

Hastings, W.K., 1970. Monte Carlo Sampling Methods Using Markov Chains and Their Applications, Biometrika, 57(1), 97-109.

Hawkins, R., Brodie, R.C. \& Sambridge, M., 2017. Trans-dimensional Bayesian inversion of airborne electromagnetic data for 2D conductivity profiles, Explor. Geophys., 49(2), 134-147.

Hubbard, A., Lawson, W., Anderson, B., Hubbard, B. \& Blatter, H., 2004. Evidence for subglacial ponding across Taylor Glacier, Dry Valleys, Antarctica, Ann. Glaciol., 39, 79-84.

Key, K., 2009. 1D inversion of multicomponent, multifrequency marine CSEM data: methodology and synthetic studies for resolving thin resistive layers, Geophysics, 74(2), F9-F20.

Key, K., 2012. Is the fast Hankel transform faster than quadrature?, Geophysics, 77(3), F21-F30.

Laine, M. \& Tamminen, J., 2008. Aerosol model selection and uncertainty modelling by adaptive MCMC technique, Atmos. Chem. Phys., 8(24), 7697-7707.

Legault, J.M., 2015. Airborne Electromagnetic Systems-State of the Art and Future Directions, CSEG Recorder, 40, 38-49.

Luo, X., 2010. Constraining the shape of a gravity anomalous body using reversible jump Markov chain Monte Carlo, Geophys. J. Int., 180(3), 1067-1079.

MacKay, D., 2003. Information Theory, Inference and Learning Algorithms, Cambridge Univ. Press.

Malinverno, A., 2002. Parsimonious Bayesian Markov chain Monte Carlo inversion in a nonlinear geophysical problem, Geophys. J. Int., 151(3), 675-688.

McGinnis, L.D. \& Jensen, T.E., 1971. Permafrost-hydrogeologic regimen in two ice-free valleys, Antarctica, from electrical depth sounding, Quat. Res., 1(03), 389-409.

Metropolis, N., Rosenbluth, A.W., Rosenbluth, M.N., Teller, A.H. \& Teller, E., 1953. Equation of state calculations by fast computing machines, $J$. Chem. Phys., 21(6), 1087-1092.

Mikucki, J.A. et al., 2015. Deep groundwater and potential subsurface habitats beneath an Antarctic dry valley, Nat. Commun., 6, 1-9.

Minsley, B.J., 2011. A trans-dimensional Bayesian Markov chain Monte Carlo algorithm for model assessment using frequency-domain electromagnetic data, Geophys. J. Int., 187(1), 252-272.
Mosegaard, K. \& Tarantola, A., 1995. Monte Carlo sampling of solutions to inverse problems, J. geophys. Res., 100(B7), 12 431-12 447.

Newman, G.A. \& Alumbaugh, D.L., 1995. Frequency-domain modelling of airborne electromagnetic responses using staggered finite differences, Geophys. Prospect., 43(8), 1021-1042.

Newman, G.A., Hohmann, G.W. \& Anderson, W.L., 1986. Transient electromagnetic response of a three-dimensional body in a layered earth, Geophysics, 51(8), 1608-1627.

Ray, A. \& Key, K., 2012. Bayesian inversion of marine CSEM data with a trans-dimensional self parametrizing algorithm, Geophys. J. Int., 191, $1135-1151$

Ray, A., Alumbaugh, D.L., Hoversten, G.M. \& Key, K., 2013. Robust and accelerated Bayesian inversion of marine controlled-source electromagnetic data using parallel tempering, Geophysics, 78(6), E271-E280.

Ray, A., Key, K., Bodin, T., Myer, D. \& Constable, S., 2014. Bayesian inversion of marine CSEM data from the Scarborough gas field using a transdimensional 2-D parametrization, Geophys. J. Int., 199(3), 18471860.

Ray, A., Sekar, A., Hoversten, G.M. \& Albertin, U., 2016. Frequency domain full waveform elastic inversion of marine seismic data from the Alba field using a Bayesian trans-dimensional algorithm, Geophys. J. Int., 205(2), 915-937.

Sambridge, M., 2013. A Parallel Tempering algorithm for probabilistic sampling and multimodal optimization, Geophys. J. Int., 196(1), 357-374.

Saygin, E. et al., 2015. Imaging architecture of the Jakarta Basin, Indonesia with transdimensional inversion of seismic noise, Geophys. J. Int., 204(2), 918-931.

Scales, J.A. \& Snieder, R., 1997. To Bayes or not to Bayes?, Geophysics, 62(4), 1045-1046.

Siemon, B., Christiansen, A.V. \& Auken, E., 2009. A review of helicopterborne electromagnetic methods for groundwater exploration, 7(1303), 629-646.

Sørensen, K. \& Auken, E., 2004. SkyTEM —a new high-resolution helicopter transient electromagnetic system, Explor. Geophys., 35, 194-202.

Steininger, G., Dettmer, J., Dosso, S.E. \& Holland, C.W., 2013. Transdimensional joint inversion of seabed scattering and reflection data, $J$. acoust. Soc. Am., 133(3), 1347-1357.

Swendsen, R.H. \& Wang, J.-S., 1987. Nonuniversal critical dynamics in Monte Carlo simulations, Phys. Rev. Lett., 58(2), 86-88.

Viezzoli, A., Christiansen, A.V., Auken, E. \& Sørensen, K., 2008. Quasi3D modeling of airborne TEM data by spatially constrained inversion, Geophysics, 73(3), F105-F113.

Yang, D. \& Oldenburg, D.W., 2012. Three-dimensional inversion of airborne time-domain electromagnetic data with applications to a porphyry deposit, Geophysics, 77(2), B23-B34.

\section{APPENDIX A: PROPOSAL DISTRIBUTION AND ACCEPTANCE PROBABILITY}

\section{A1 Initialization}

Initializing the trans-dimensional MCMC algorithm is simple. We select an initial model for the ensemble by setting $k=k_{\min }$. In our case, $k_{\min }=1$, so our model has one interface and two layers. We select the interface depth and layer resistivities uniformly within the prior bounds.

\section{A2 Likelihood}

In this section we discuss our choice for the form of the likelihood function, $p(\mathbf{d} \mid \mathbf{m})$. We use a simple Chi-squared $\left(\chi^{2}\right)$ framework measure of misfit

$\chi^{2}=(\mathbf{d}-\hat{\mathbf{d}}) \mathbf{C}_{d}^{-1}(\mathbf{d}-\hat{\mathbf{d}})$ 
where $C_{d}^{-1}$ is the matrix of data covariances and $\hat{\mathbf{d}}$ is the modelled data: $f(\mathbf{m})=\hat{\mathbf{d}}$ and $f$ is the forward modelling function. This choice of misfit corresponds to a Gaussian likelihood function

$p(\mathbf{d} \mid \mathbf{m})=\exp \left(\frac{-\chi^{2}}{2}\right)$.

\section{A3 Prior}

In this section we discuss the prior, $p(\mathbf{m})$, in more detail. In principle, the prior represents all known information about the model that is independent of the measured data, but in practice this information must be expressed in terms of a probability distribution. Because our framework is trans-dimensional - the number of unknowns is allowed to vary - the prior is actually a function of $\mathbf{m}$ and $k$ and can be written in two parts

$p\left(\mathbf{m}_{k}, k\right)=p\left(\mathbf{m}_{k} \mid k\right) p(k)$

where the first term of the right hand side is the prior on $\mathbf{m}$ given a fixed number of layer interfaces, $k$. The second term on the right hand side is the prior on the number of interfaces. Since we have little idea beforehand how many parameters the data will require, we choose for $p(k)$ a uniform distribution with generous bounds:

$p(k)=\left\{\begin{array}{cl}\frac{1}{k_{\max }-k_{\min }+1} & \text { if } k_{\min } \leq k \leq k_{\max }, \quad k \in \mathbb{N} \\ 0 & \text { else. }\end{array}\right.$

To ensure that $k_{\max }$ and $k_{\min }$ were sufficiently generous, the posterior distribution on $k, p(k \mid \mathbf{d})$, can be examined after the Bayesian inversion has run to completion. The overwhelming bulk of the models should fall away from the edges of the distribution - otherwise, a wider prior may be needed.

There is some correspondence between the prior and a reference model used in regularized inversions, if the latter represents $a$ priori knowledge of the model. The analogy, however, can be misleading. For instance, while a reference model is used to keep each model parameter from straying too far from its reference value, a uniform prior assumes all values are equally likely for each model parameter.

Importantly, the prior is agnostic as to the units of the various model parameters, which are not required to have the same unitse.g. interface depth $(\mathrm{m})$ and layer resistivity $(\mathrm{ohm} \mathrm{m})$. This is because the prior for each parameter is expressed as a probability.

The prior on the model, given a fixed number of interfaces (and hence layers as well), must now be determined. While we expect some correlation in the posterior distribution between the depths of layers and their electrical resistivity, we do not know the form this correlation will take, a priori, so we assume that the prior distributions on the depths of interfaces and the resistivities of the layers are independent, enabling us to write

$p\left(\mathbf{m}_{k} \mid k\right)=p(\mathbf{z} \mid k) p(\rho \mid k)$.

The conditional prior distribution on the layer interface locations, $p(\mathbf{z} \mid k)$ is determined by first deciding that each of the $k$ layer interfaces must lie within a depth range, $\Delta z$, and that all locations within that depth range are equally likely to host an interface. Then, we recognize that $p(\mathbf{z} \mid k)$ is Dirichlet distributed, leading to

$p(\mathbf{z} \mid k)= \begin{cases}\frac{k !}{\Delta z^{k}} & \text { if } z_{\min } \leq z_{i} \leq z_{\max } \quad \forall i=1,2, \ldots k \\ 0 & \text { else. }\end{cases}$

The Dirichlet distribution describes how a unit interval is divided into $k$ parts, and can be explained fairly simply: given $k$ interfaces, each distributed independently and uniformly over a depth interval $\Delta z$, there are $k$ ! ways of arranging those interfaces among the selected depths. This yields eq. (A6). This prior is first used in Steininger et al. (2013) and derived in detail in Dosso et al. (2014).

No prior knowledge of the resistivity, $\rho$, is assumed, only that it must lie in some bounded interval $\left(\rho_{\min }, \rho_{\max }\right)$. Because no known prior correlation is assumed between the resistivity of the $k$ layers, the layer properties prior has the simple form

$p(\rho \mid k)= \begin{cases}\frac{1}{\Delta \rho^{k+1}} & \text { if } \rho_{\min } \leq \rho_{i} \leq \rho_{\max } \quad \forall i=1,2, \ldots k+1 \\ 0 & \text { else }\end{cases}$

where $\Delta \rho=\rho_{\max }-\rho_{\min }$.

Finally, combining eqs (A3)-(A7), we obtain the full prior distribution for $k$ interfaces and $k+1$ layers:

$p\left(\mathbf{m}_{k}, k\right)=\frac{k !}{\Delta k \Delta \rho^{k+1} \Delta z^{k}}$

where $\Delta k=k_{\max }-k_{\min }+1$.

\section{A4 Proposal distributions and acceptance probabilities}

There is significant freedom in the choice of proposal distribution. In theory, convergence of the Markov chain to the posterior is agnostic of the choice of $q\left(\mathbf{m}^{\prime} \mid \mathbf{m}\right)$ : the proposal only affects the rate of convergence, not the guarantee of convergence itself. In practice, however, computational cost is of paramount concern in Bayesian methods, so making an effective choice of proposal distribution is important. In our implementation of trans-dimensional MCMC there are four ways to perturb the last model in the chain to produce a proposal model: update, move, birth, and death. These moves are summarized, along with their proposal distributions and acceptance probabilities, in the following sections:

(i) A move shifts the location of an interface. The new location is drawn from a Gaussian distribution centred at the current interface location. If this new location would place the interface above or below its nearest neighbours, the resistivity of the affected layers are treated as if this perturbation were the same as a death followed by a birth. This perturbation is chosen with probability $6 / 32$.

(ii) An update does not change the dimensionality of the model. Instead, we perturb the resistivity of each layer about its mean by drawing from a Gaussian distribution. This kind of perturbation is chosen with probability 16/32.

(iii) A birth adds a layer interface at a random depth chosen uniformly in the interval $\left[\begin{array}{ll}z_{\min } & z_{\max }\end{array}\right]$. The resistivity of the new layer either above or below the new interface is drawn from a Gaussian distribution, centred at the resistivity of the layer prior to the perturbation. This perturbation is chosen with probability 5/32.

(iv) A death deletes one randomly selected layer interface. The resistivity of the layer above or below the deleted interface is chosen and applied to the new, larger layer. This perturbation is chosen also with probability 5/32 (to ensure births and deaths are equally likely).

The acceptance probability $\alpha$ depends on the proposal ratio (see eq. 3), not on the proposal directly, so the proposal ratios for each move must be calculated.

\section{A4.1 Update and move interface}

For moves of fixed dimension (the number of model parameters does not change), such as the update and move interface perturbations, this is simple. We have chosen symmetric distributions (Gaussian and uniform) governing these moves, so the move from $\mathbf{m}$ to $\mathbf{m}^{\prime}$ 
should be equally as likely as a move from $\mathbf{m}^{\prime}$ to $\mathbf{m}$. Likewise, the prior is identical for fixed $k$ (see Equation A8). This leads to a simple form for the acceptance probability

$\alpha\left(\mathbf{m}^{\prime} \mid \mathbf{m}\right)_{\text {fixed dimension }}=\min \left[1,\left(\frac{p\left(\mathbf{d} \mid \mathbf{m}^{\prime}\right)}{p(\mathbf{d} \mid \mathbf{m})}\right)^{1 / T}\right]$

which, for $T=1$, is simply the ratio of the likelihoods. This, of course, assumes that all the parameters within $\mathbf{m}^{\prime}$ fall within the prior bounds for the allowed depth of interfaces and the bounds on electrical resistivity.

\section{A4.2 Birth}

Because the selection of the new interface location and the selection of the new resistivity are independent, we can write for the proposal

$q\left(\mathbf{m}^{\prime} \mid \mathbf{m}\right)_{\text {birth }}=\frac{1}{\Delta z \sqrt{2 \pi} \sigma_{\text {birth }}} \exp \left[-\frac{\left(\rho^{\prime}-\rho\right)^{2}}{2 \sigma_{\text {birth }}^{2}}\right]$.

The reverse of a birth (going from $\mathbf{m}^{\prime}$ to $\mathbf{m}$ ) is a death. In this case, there are $k+1$ interfaces to choose from ( $\mathbf{m}^{\prime}$ has $k+1$ interfaces), so the proposal ratio for a birth is

$$
\left[\frac{q\left(\mathbf{m} \mid \mathbf{m}^{\prime}\right)}{q\left(\mathbf{m}^{\prime} \mid \mathbf{m}\right)}\right]_{\text {birth }}=\frac{\Delta z \sqrt{2 \pi} \sigma_{\text {birth }}}{k+1} \exp \left[\frac{\left(\rho^{\prime}-\rho\right)^{2}}{2 \sigma_{\text {birth }}^{2}}\right] .
$$

Meanwhile, the prior ratio for a birth is

$\left[\frac{p\left(\mathbf{m}^{\prime}\right)}{p(\mathbf{m})}\right]_{\text {birth }}=\frac{k+1}{\Delta z \Delta \rho}$.

So the final acceptance probability for a birth is given by

$\alpha\left(\mathbf{m}^{\prime} \mid \mathbf{m}\right)_{\text {birth }}=\min \left[1,\left(\frac{p\left(\mathbf{d} \mid \mathbf{m}^{\prime}\right)}{p(\mathbf{d} \mid \mathbf{m})}\right)^{1 / T} \frac{\sqrt{2 \pi} \sigma_{\text {birth }}}{\Delta \rho} \exp \left[\frac{\left(\rho^{\prime}-\rho\right)^{2}}{2 \sigma_{\text {birth }}^{2}}\right]\right]$.

\section{A4.3 Death}

A death deletes one randomly selected layer interface. The resistivity of the layer above or below the deleted interface is randomly chosen and applied to the new, larger layer. This perturbation is chosen also with probability 5/32 (to ensure births and deaths are equally likely).

Since we can remove any of $k$ available layers, the proposal for a death move is

$q\left(\mathbf{m}^{\prime} \mid \mathbf{m}\right)_{\text {death }}=\frac{1}{k}$.

The reverse is a birth move. This means that the proposal ratio for a death is given by

$$
\left[\frac{q\left(\mathbf{m} \mid \mathbf{m}^{\prime}\right)}{q\left(\mathbf{m}^{\prime} \mid \mathbf{m}\right)}\right]_{\text {death }}=\frac{k}{\Delta z \sqrt{2 \pi} \sigma_{\text {birth }}} \exp \left[-\frac{\left(\rho^{\prime}-\rho\right)^{2}}{2 \sigma_{\text {birth }}^{2}}\right] .
$$

Meanwhile, the prior ratio for a death is given by

$\left[\frac{p\left(\mathbf{m}^{\prime}\right)}{p(\mathbf{m})}\right]_{\text {death }}=\frac{\Delta z \Delta \rho}{k}$.

So the final acceptance probability for a death is

$$
\begin{aligned}
\alpha\left(\mathbf{m}^{\prime} \mid \mathbf{m}\right)_{\text {death }}= & \min \left[1,\left(\frac{p\left(\mathbf{d} \mid \mathbf{m}^{\prime}\right)}{p(\mathbf{d} \mid \mathbf{m})}\right)^{1 / T} \frac{\Delta \rho}{\sqrt{2 \pi} \sigma_{\text {death }}}\right. \\
& \left.\times \exp \left[-\frac{\left(\rho^{\prime}-\rho\right)^{2}}{2 \sigma_{\text {death }}^{2}}\right]\right]
\end{aligned}
$$

\section{APPENDIX B: TRANS-DIMENSIONAL MCMC PSEUDO-CODE}

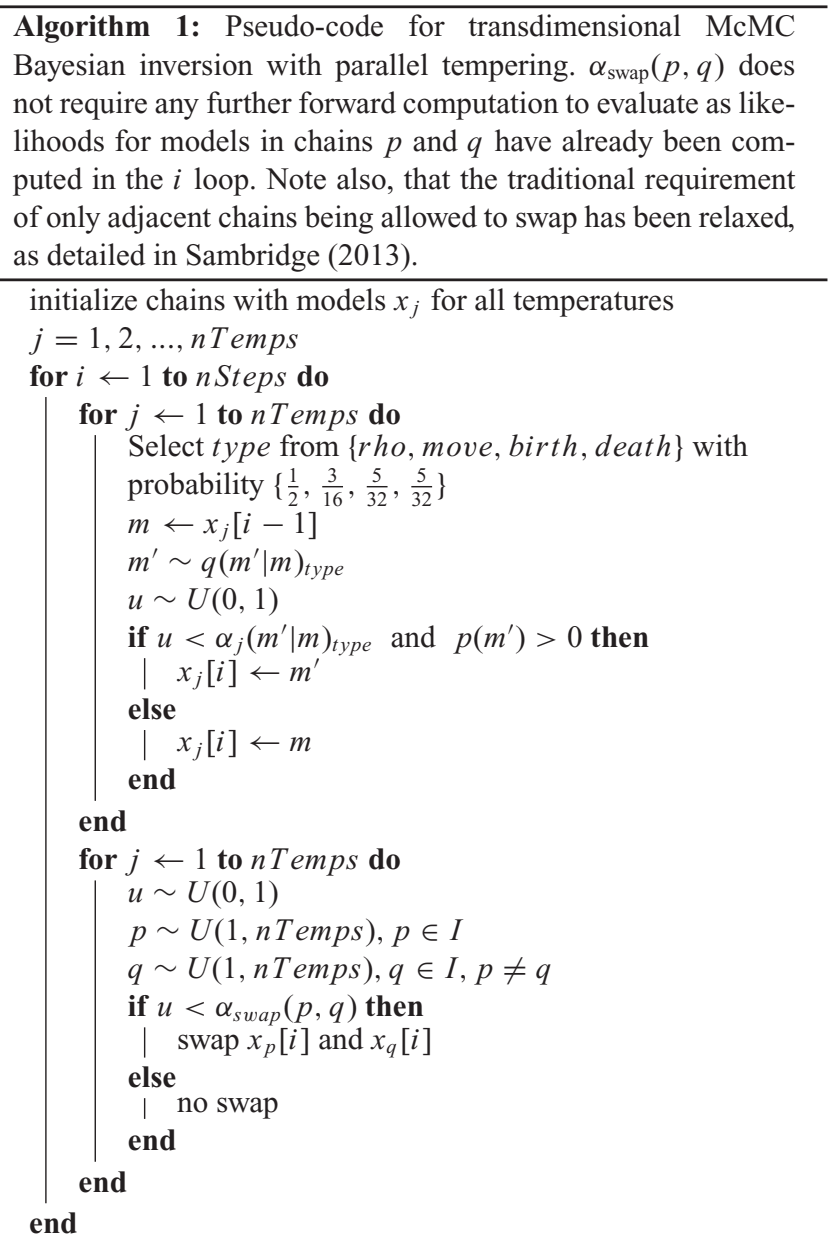

